\title{
VELOCITY FIELD MEASUREMENTS DURING DROP FORMATION BY VORTICAL FLOWS IN MICROGRAVITY
}

\author{
L.P. Bernal ${ }^{*}$ and P. Maksimovic ${ }^{\dagger}$ \\ Department of Aerospace Engineering \\ University of Michigan \\ Ann Arbor, MI. 48109-2140
}

\begin{abstract}
$\underline{\text { Abstract }}$
We report velocity field measurements using Particle Image Velocimetry (PIV) of the interaction of a vortex ring with the interface between immiscible liquids. To obtain high resolution PIV images, the refractive index of the two liquids were matched. In addition the density change across the interface was small resulting in surface-tension-dominated interface dynamics. For these experiments the range of Weber number studied is high enough to result in interface breakup and drop formation. The velocity field measurements are processed to determine the evolution of the vorticity field during the interaction and drop formation process. We show that at low Weber number large amounts of opposite sign vorticity are generated at the interface. This vorticity diffuses across the interface as well as into the vortex ring. In contrast at higher Weber numbers the vortex ring interaction occurs in a shorter time scale and does not result in generation of opposite sign vorticity. However the circulation of the vortex ring is reduced during the interaction.
\end{abstract}

\section{$\underline{\text { Introduction }}$}

The interaction of a vortex ring with immiscible fluid interfaces captures the interplay between inertia, interface tension, viscous and gravitational forces that can be found in a variety of flow problems. Of

\footnotetext{
* Associate Professor, Senior Member AIAA.

$\dagger$ Present address: Allied Signal, Tempe, AZ.
}

Copyright (C) 1998 by Luis P. Bernal. Published by the American Institute of Aeronautics and Astronautics, Inc. with permission particular interest is the non-linear response and evolution of the fluid interface under the perturbations introduced by the vortex. The interaction captures the essential dynamics associated with flow-induced breakup of the interface and drop formation processes. Yet, at the same time, this problem is a relatively simple flow configuration that can be studied both experimentally and numerically.

The flow configuration is illustrated schematically in Figure 1. A vortex ring is produced by a generator at the bottom of a test cell. The vortex ring propagates upward in the lower liquid layer as shown in Figure 1 (a). The vortex ring reaches the interface and, if it has sufficient momentum, it causes break up of the interface and formation of a drop of liquid from the lower layer in the upper liquid layer, as illustrated in Figure 1 (b). The parameters that characterize the interaction are: the circulation of the vortex ring, $\Gamma$; the diameter of the vortex ring, $a$; the interfacial tension, $\sigma$; the density of the liquids, $\rho_{1}$ and $\rho_{2}$; and the viscosity of the liquids, $\mu_{1}$ and $\mu_{2}$. In addition for earth based experiments the acceleration of gravity, $\mathrm{g}$, is also an important parameter. The relevant nondimensional numbers that characterize the flow are: the Weber number, We; the Bond number, Bo; and the Reynolds number, Re. They are defined as follows:

$$
\begin{aligned}
& \mathrm{We}=\frac{\rho_{1} \Gamma^{2}}{\sigma a} \\
& \mathrm{Bo}=\frac{\left(\rho_{1}-\rho_{2}\right) \mathrm{g} a^{2}}{\sigma} \\
& \operatorname{Re}=\frac{\rho_{1} \Gamma}{\mu_{1}}
\end{aligned}
$$


Also the density ratio, $r=\rho_{2} / \rho_{1}$, and the viscosity ratio, $\quad \nu=\mu_{2} / \mu_{1}$, are important. Another nondimensional parameter frequently used in atomization studies is the Ohnesorge number, Oh, defined as

$$
\mathrm{Oh}=\frac{\mathrm{We}}{\mathrm{Re}}=\frac{\mu_{1}}{\sqrt{\rho_{1} \sigma a}}
$$

In microgravity experiments the range of parameters of interest is We $\gg 1$ for interface breakup, Bo $<<1$, with $r \ll 1, v \ll 1$. In typical atomization systems We $\gg 1$, Bo $<1$, and $r \ll 1, v \ll 1$. The Reynolds number and Ohnesorge number vary considerable depending on the viscosity of the liquid. For the present experiments using two liquid layers of almost matched density, the density and viscosity ratio are of order one (i.e. $r \sim 1, v \sim 1$ ).

Bernal et al ${ }^{1}$ report flow visualization experiments and numerical simulations of the interaction of a vortex ring with an immiscible liquid interface. The results showed very good agreement between the flow visualization results and the numerical results. However because of the nature of the flow visualization data, the comparisons were limited to a few geometrical features of the flow. More detailed comparisons of the flow evolution were not possible. In particular velocity field data are essential to validate the more subtle aspects of the computational procedure.

To address the limitations of the Bernal et al ${ }^{1}$ data, the present experimental program was undertaken. PIV velocity field data was obtained at several times during the interaction of a vortex ring with an immiscible liquid interface. The PIV data was processed to obtain the vorticity filed during the interaction. Finally we report measurements of the maximum vorticity and circulation of the various vorticity containing regions in the flow.

\section{Flow Apparatus and Measurement}

\section{Techniques}

\section{Flow Apparatus}

The experiments were carried out in a liquid container of inner dimensions $11 \times 11 \times 30 \mathrm{~cm}$. These values were chosen as a compromise between minimizing end-wall effects on the propagation of the vortex ring, and minimizing the cross-section linear dimension for the PIV measurements as well as the amount of liquid used in the tests. The walls of the container were made of a transparent material, thus allowing the flow to be viewed from all four sides. The bottom of the container was a brass base-plate assembly which houses the vortex ring generator. The vortex ring generator consists of a pneumatically-driven piston, whose upward motion produces the vortex rings. A solenoid valve and a pressure regulator control the intake air. The generator wall has four small orifices used to inject dye for flow visualization experiments.

The diameter, $D_{p}$, and the stroke, $L_{p}$, of the piston are $1.27 \mathrm{~cm}$. The choice of $\mathrm{L}_{\mathrm{p}} / \mathrm{D}_{\mathrm{p}}=1$ was motivated by the requirement of achieving the highest Reynolds number possible for a laminar vortex ring. The results of Glezer ${ }^{2}$ show that laminar vortex rings are formed up to Re $-5000-10,000$ for $L_{p} / D_{p}=1$. Other values of $L_{p} / D_{p}$ result in early transition of the vortex ring which would limit the range of conditions that can be tested in the apparatus.

The strength of the vortex ring and the corresponding propagation speed was varied by changing the pressure of the intake air. The motion of the piston during the stroke was monitored by an infrared detector. The position of the piston was recorded on a digital oscilloscope and used to measure the duration of the stroke, thus the average speed of the piston motion could be determined. At the end of motion, the piston surface was flush with the bottom surface of the liquid container. For the range of intake air pressures of 10 to $60 \mathrm{psig}$, the time to complete the full $1.27 \mathrm{~cm}$ stroke varied from 6 to $60 \mathrm{~ms}$, which corresponds to $10-100 \mathrm{~cm} / \mathrm{s}$ average piston velocity.

At each experimental condition (i.e., input air pressure), several realizations were conducted. Tests showed that in order to obtain reproducible vortex ring motion, the minimum elapsed time between runs had to be about a minute, thus allowing the fluid motion in the liquid container to stop, and the interface to stabilize.

\section{PIV Technique}

The PIV system used in this study is a film-based system, employing two Nd-YAG lasers to illuminate the flow and an oscillating mirror for velocity bias. The PIV images were scanned and digitally processed using a cross correlation technique to determine the velocity on the entire flow region recorded in the images. PIV was used in two types of experiments. First, it was used 
to characterize the vortex ring motion without an interface. These results were further used to validate the PIV data acquisition and processing procedures. The results of these experiments will not be described here. They can be found in Ref. 3. Second, the PIV technique was used to measure the velocity field for the case of a single interface. For these tests the refractive index of the two liquids were matched to achieve the required optical resolution. These results are the focus of the present paper

\section{PIV Image Acquisition}

A schematic diagram of the PIV imaging system is shown in Figure 2. Two Nd-YAG lasers (Spectra Physics GCR-11) are used to form sheets of green (532 $\mathrm{nm}$ ) light in the plane of symmetry of the flow. The duration of each light pulse is $6 n s$. The sheet forming optics consists of a spherical and a cylindrical lens. The thickness of the sheet was $150 \mu \mathrm{m}$. Because two different lasers are used, it was necessary to adjust the overlap the two laser light sheets. A procedure was developed to adjust the laser sheet overlap with a resolution better than $1 / 8^{\text {th }}$ of the laser sheet thickness. The two lasers were triggered sequentially. The time lapse between lasings was adjusted by a delay generator (Tektronix PFG 51005) and the actual time lapse was measured by a counter (HP 5334A). The prescribed time delay varied between $60 \mu \mathrm{s}$ to $6 \mathrm{~ms}$, inversely proportional to the expected maximum flow velocity. The actual value was adjusted to insure a particle displacement on the film consistent with the spatial resolution and accuracy of the measurement.

In the present PIV system the two flow images are recorded on the same photographic film. This simplifies the photographic recording system but introduces velocity direction ambiguity since it is not possible to differentiate between the first and second exposure of the particles. A number of approaches for resolving the directional ambiguity of PIV have been proposed including: electrooptical image shifting based on differing polarization of the laser light, ${ }^{4}$ and flow recording on physically separate frames, such as in video-based cross-correlation, or DPIV. ${ }^{5}$

To resolve directional ambiguity, as well as to provide control over the range of particle displacements in the image, a bias velocity in the direction perpendicular to the vortex ring motion was introduced in the present experiments. The image shifting technique first proposed by Adrian $^{6}$ was used. An oscillating mirror (General Scanning G325DT) positioned in front of the camera lens shifted the flow image between laser firings. The amplitude and direction of the bias velocity is determined by the amplitude and frequency of the scanning mirror motion which was varied from $\pm 0.019 \mathrm{rad}$ to $\pm 0.095 \mathrm{rad}$ at a frequency of $10 \mathrm{~Hz}$. The bias velocity was always well above the maximum velocity of the vortex ring flow, thus the flow direction was uniquely determined. The PIV images were recorded with a $35 \mathrm{~mm}$ SLR camera (Nikon FM) equipped with a motor drive, bellows and a $135 \mathrm{~mm}$ Nikkor lens. Kodak Tmax -400 film was used in all the tests reported here. Film to flow magnification was $1: 2$.

A critical aspect of the PIV measurements is synchronization of the various components of the system (oscillating mirror, camera shutter, lasers) and the vortex ring generator. The timing signals for a typical experiment are shown in Figure 3. The oscillating mirror and the lasers' flashlamp were operated continuously at the operating frequency of the lasers $10 \mathrm{~Hz}$. This insured thermal equilibrium of the Nd-YAG crystal and, therefore, good optical performance. Further, the oscillating mirror and the lasers must be operated in phase to insure that the mirror is at the same angular position when the lasers are fired and the PIV images are recorded. This was accomplished using a common $10 \mathrm{~Hz}$ sine wave signal produced by an external signal generator (HP 8904A) to operate the mirror and the lasers. This signal was fed directly to the oscillating mirror controller and to a pulse generator (Syston Donner 100C). The pulse generator output, labeled "Master Clock" in the schematics, is a square wave signal delayed with respect to the sinusoidal wave so that the PIV images are recorded at the point of maximum angular velocity of the mirror. The pulse generator output is further delayed by a second pulse generator (Tektronix PFG 51005) and its output used to trigger the flashlamp of the second laser as described above.

Although these signals provide synchronization between the lasers and the oscillating mirror, actual timing for the images is controlled by a specially designed circuit, labeled "Synch Electronics" in Figure 3. The "Synch Electronics" box controls the operation of the vortex ring generator, the camera shutter and the Q-Switch trigger of the lasers. Input to the "Synch Electronics" box are the Master Clock, time delay between the vortex ring generator motion and laser firing, as well as the manual trigger command to initiate the process. The "Synch Electronics" box generates the necessary timing signals to initiate the vortex ring 
generator motion, to trigger the $35 \mathrm{~mm}$ SLR camera, and to fire the lasers, as indicated in Figure 3. Thus when the "manual trigger" on the "trigger box" was manually operated, the piston of the vortex ring generator fired, producing a vortex ring. The camera shutter opened, and the lasers fired at the prescribed time delay. The shutter stayed open for approximately $250 \mathrm{~ms}$, and upon its closing, the motordrive wound the film to the next frame.

\section{$\underline{\text { Seeding }}$}

In the present experiments the flow was seeded with silver-coated hollow glass spheres (Potters Industries) of $14 \mu \mathrm{m}$ mean diameter. The Scott apparent density of the spheres was $0.496 \mathrm{~g} / \mathrm{cm}^{3}$. Due to a substantial variance in the weight distribution of the particles, filtration was needed to remove the smallest and the largest particles and prevent excessive settling and accumulation of seed particles at the liquid interface during the duration of the experiments.

The concentration of seed particles was optimized for best performance of the processing software. Adrian ${ }^{7}$ suggests a concentration of at least 10 particles per interrogation volume. This value is consistent with our results.

\section{Index of Refraction Matching}

To accurately measure the velocity field across multiple liquid layers, good optical resolution across the interface is required. For liquid layers having different refractive properties, the optical path of the light scattered from the seeded flow is distorted across the interface, causing a blur in the image. Thus, to prevent this distortion, the refractive indices of the liquids need to be matched. The method used is based on adding various solutes in different proportions into a liquid in order to modify the refractive index, as well as its density. ${ }^{8-10}$

For the purpose of the present study, there were three requirements for a proper solvent. First, in order to eliminate optical distortions, the refractive index of the solution had to match the refractive index of the other liquid layer. Second, the liquid layers had to be immiscible in order to have an interface. And, third, the density change across the interface had to be small in order to examine flow at the capillary limit (surfacetension-dominated interface dynamics).
The liquid pair which fulfilled all three requirements was a mixture of propyl alcohol and propyl glycol in distilled water, and silicone oil. The volumetric mixture fraction of water to glycol to alcohol was, approximately, 1:2:2.5. Silicone oil of $10 \mathrm{cS}$ viscosity formed the upper layer. The resulting index of refraction of the water-based mixture matched the index of refraction of the silicone oil. At the same time, the density difference between the water-based solution and the silicone oil was smaller than $0.2 \%$. While the propyl glycol increased the refractive index of the mixture, the propyl alcohol decreased the mixture density. The addition of water rendered the solvent immiscible with the oil, and thus prevented diffusion across the interface. Tests were conducted to confirm that diffusion across the interface did not occur.

\section{PIV Data Processing}

The PIV images were digitized with a slide scanner (Nikon Coolscan model LS-10E) at 2700 pixels per inch $(=9.4 \mu \mathrm{m} /$ pixel) resolution. The PIV processing software determined the velocity at points on a equally spaced grid covering the region of interest in the flow. The spacing between points on the grid was 16 pixels, or $0.3 \mathrm{~mm}$ for a magnification of $1: 2$. The particle displacement field was determined using a crosscorrelation technique applied to an interrogation window around the measurement point. ${ }^{6,11,12}$ Spatial cross-correlation between displaced windows was used. The size of the interrogation window was $64 \times 64$ pixels, corresponding to $1.2 \times 1.2 \mathrm{~mm}$ in the flow, for a magnification of $1: 2$. The particle displacement was determined as the maximum of the cross-correlation function within a suitably chosen search window. If the maximum value of the correlation was found on the boundary of the search window the result was assumed to be invalid. A typical PIV image was interrogated at 12,000 to 30,000 points over the entire region of interest. Of all these displacement vectors not more than $2 \%$ were found to be invalid. The invalid data points were removed with a median filter which also removed outliers. Once the displacement vector within each interrogation window was determined, velocity vectors were readily obtained by dividing the displacement by the time lapse between the two laser firings.

The vorticity was computed from the velocity data using Stokes theorem. The circulation around each point was calculated using 8 neighboring points. The line integrals required for the calculation of the circulation were evaluated using Simpson's rule. 
The final step in the data processing was application of a low pass filter in order to remove noise of high spatial frequency. The data was processed by a lowpass filter with passband frequency ( $-3 \mathrm{~dB}$ point) of $3.32 \mathrm{~cm}^{-1}$ (wavelength $3 \mathrm{~mm}$ ) and stopband $(-\infty \mathrm{dB})$ frequency of $6.64 \mathrm{~cm}^{-1}$ (wavelength $1.5 \mathrm{~mm}$ ). The relatively low cut-off frequency is justified by the low Reynolds number of the tests. The parameters of the filter were validated with measurements on a vortex ring flow. ${ }^{3}$

\section{Measurement Uncertainty and Accuracy}

The uncertainty of the PIV measurements has been discussed by several authors. ${ }^{11-15}$ Most of the work reported in the literature addressing PIV data validation, noise reduction, or accuracy, has been based either on theoretical analysis or on analysis of numerical simulations, thus providing mostly a general guidance of the requirements to be fulfilled in order to obtain a good quality images and the effect of errors. This procedure was considered inadequate for the present investigation because the effect of residual flow motions in the tank on the measurement uncertainty could not be quantified. Thus, in the present investigation, the measurement uncertainty was determined directly by measurements of the flow field with the fluid in the tank at rest. These PIV data were also used to determine the actual bias velocity. The results showed no particular trend of the data that could result from optical distortion. Thus all the measured velocities can be considered independent realizations of a field of constant velocity equal to the bias velocity.

Typical histograms of the measured particle displacement components and the computed vorticity are presented in Figure 4. The width of the histograms illustrates the basic uncertainty of the measurements. For the histograms shown in Figure 4, the standard deviations are 0.218 pixels, 0.164 pixels, and 0.007 for the $\mathrm{x}$ - displacement component, $\mathrm{y}$ - displacement component and vorticity respectively. Because the time lapse between exposures varied, the actual uncertainty changed between different realizations. This uncertainty is believed to be determined by the particle size and the number of particles in the interrogation window.

The effect of velocity gradients within the interrogation window on the measurement uncertainty was found to be negligible. For these experiments the change in displacement within the interrogation window due to velocity gradients is small compared with the particle diameter. Therefore, its effect on signal to noise ratio of the cross-correlation function is negligible. This was confirmed by a very small number of bad data points obtained during image processing. The bad data points were randomly distributed and did not correlate with the location of the vortex cores.

The effect of filtering on measurement uncertainty is illustrated in the histograms presented in Figure 5. The standard deviations after filtering are 0.083 pixels, 0.055 pixels, and 0.001 , for the $x$ velocity component, $y$ velocity component and vorticity respectively. These values are representative of the actual uncertainty of the present PIV velocity measurements. The actual values varied slightly between different realizations. An upper bound of the measurement uncertainty is reported in the sections below.

\section{Physical Properties of the Liquids}

As described above the container was filled with two liquid layers. Immiscible liquid pair combinations were formed using silicone oil and water-based mixtures. Several silicone oils with varying viscosity were used in the tests. The density, viscosity and refractive index of the water mixture was adjusted by varying the composition of the mixture to match the refractive index of the silicone oil. Several liquids were used including methyl alcohol, propyl alcohol, and propylene glycol.

The following physical properties of the liquids were measured: density, index of refraction, interfacial tension, and viscosity. Liquid density was measured using Fisher Scientific hydrometers with an accuracy of $0.001 \mathrm{~g} / \mathrm{cm}^{3}$. The index of refraction was measured with the Atago N2 hand-held refractometer, with the measuring span of $28-62 \%$ Brix scale; the error of the measurement was $0.1 \%$. A DG $24 / 27$ controlled strain rheometer was used to measure the viscosity of the liquids, with an accuracy of $5 \%$. The surface tension of the distilled water was measured by the ring method, using a Fisher Surface Tensiomat, model 21. Interface tension of the liquids of similar densities was measured by the capillary-rise method described by Karri and Mathur. ${ }^{16}$ A glass apparatus with a capillary leg was specially designed and fabricated for this purpose. According to Karri and Mathur, ${ }^{16}$ the accuracy of the method is better than $10 \%$.

\section{$\underline{\text { Results and Discussion }}$}

PIV measurements were obtained at several flow conditions. The interface properties are given in Table 
1. The Weber number was varied in the range 125 1100. The critical Weber number for the interface was 125. No drops formed for Weber numbers less than 125.

\section{Critical Weber Number Case}

Figure 6 shows a sequence of PIV velocity (right) and vorticity (left) data for $\mathrm{We}=125$. The velocity vector field and vorticity contours are overlaid on the actual PIV image showing the location of the interface. The interface is visualized by the change in image contrast resulting from different seed particle concentration in the two layers. The time shown for each case is measured from the initiation of the vortex ring generator motion. The sequence of PIV data (a)(d) shows the deformation of the interface caused by the vortex ring and formation of the liquid column. The vorticity contours show highly concentrated vorticity at the location of the vortex ring, and formation of a nodule around the concentrated vorticity. As a liquid column forms below the nodule, vorticity is generated at the interface in the high curvature regions right under the nodule, (b)-(c). The contours in these images clearly show diffusion of vorticity beyond the interface. This thin layer of vorticity remains attached to the nodule surface up to $t=0.857 \mathrm{~s}$. In subsequent images, (d) to (f), there is separation of the boundary layer on the nodule and vorticity is shed away from the necking column. The velocity field shows the formation of a stagnant flow region in the lower part of the liquid column, (d)(e), and generation of vorticity at the interface in the region of high interface curvature at the bottom of the column. Note that the vorticity generated by the high surface curvature is of opposite sign compared to the vortex ring vorticity on the same side of the symmetry axis. There is also some vortex ring vorticity at the bottom of the column shed from the vortex ring as it propagated through this region.

As the vortex ring motion continues, the liquid column stretches and the upward velocity is reduced. Downward velocity at the bottom of the liquid column is observed in images (g) and (h). The vorticity generation at the interface is very strong in this region. The distribution of vorticity shows that the vorticity maximum coincides with maximum curvature of the interface, which suggest that vorticity is generated by the motion of the fluid inside the column along the highly curved interface. The motion of low density fluid near the top of the column stops at $t=1.477 \mathrm{~s}$, and downward motion follows. The maximum elevation of the interface is reached some time between images ( $g$ ) and (h). The vorticity distribution shows a reduction of the vortex ring vorticity and cancellation of vorticity at the interface. The vorticity generation at the interface is attributed to unsteady rotation and is maximum at the point on the interface farther from the axis in image (h). The location of the maximum vorticity moves towards the top of the liquid column as the downward motion inside the column develops (i)-(l). Interface breakup occurs at the bottom of the column (i). Large values of the vorticity are measured at the pinch-off point, as well as a fairly large downward velocity. After interface breakup, the bottom of the column pulls up by interfacial tension while the interface on top of the lower layer continues moving downward, $(j)-(k)$. The last image in Figure 6 shows a more dense liquid drop formed in the upper layer. The shape of the drop is ellipsoidal. The vorticity distribution has two welldefined peaks located outside the drop and the flow velocity is downward inside the drop.

\section{High Weber Number Case (We $=1085)$}

Increase of the Weber number changes the location of interface breakup. Instead of pinch-off at the bottom of liquid column, the interface breaks beneath the nodule carrying the vortex ring. This type of interaction is illustrated by the $\mathrm{We}=1085$ case.

Photographic PIV images in Figure 7 show snapshots of the interaction, and capture characteristic features of the flow evolution. Similar to previous Weber number cases, the vortex lifts the interface and creates a liquid column. However, due to increased inertia, the shape of the vortex cross-section now changes very little through the interaction. Also, the vorticity shedding to the liquid column is very small. Thus, an elliptically shaped nodule atop the liquid column appears. Furthermore, the column itself has a different shape. The column is very wide at its base and gradually tapers toward the top. The neck of the column is beneath the vortex nodule. The upward motion of the vortex in the upper layer results in interface breakup at the neck and a vortex drop is generated.

\section{Maximum Vorticity and Circulation}

Several regions of concentrated vorticity, labeled primary, secondary and tertiary vortex, can be identified in the flow evolution shown in Figure 6. The maximum vorticity and the total circulation associated with each of these vortices were measured using the PIV vorticity data. The results for the evolution of the maximum vorticity of the three vortices and the drop are presented 
in Figure 8 . The total circulation in these regions is plotted in Figure 9. The letter next to each symbol identifies the corresponding image in Figure 6.

The data in Figure 8 show that for the vortex ring just above the interface, (a), the vorticity is already reduced by approximately $1 / 4$. Further propagation of the ring into the upper layer results in a continuous reduction of the vorticity of the ring, until complete cancellation. The process of vorticity attenuation is relatively rapid. Simultaneously with ring vorticity attenuation, the net value of the counter-rotating vorticity of the secondary vortex increases, reaching a peak value $1 / 6^{\text {th }}$ of the maximum vorticity of the original vortex ring. Increased necking of the liquid column intensifies generation of the tertiary vorticity having the same sign as the primary vortex ring, thus causing a reduction of the secondary counter-rotating vorticity. This new, highly localized, tertiary vorticity grows rather quickly. Upon interface breakup, the vorticities of both the newly created tertiary vortex and the drop are approximately $1 / 8$ of the initial maximum vortex ring vorticity.

Figure 9 shows the rate of change of circulation of the primary vortex ring, the secondary vortex, the tertiary vortex, and the vortex drop. The circulation was evaluated by integrating the vorticity enclosed in the vorticity contour value of $10 \%$ of the peak vorticity. The letters along the data lines indicate the corresponding PIV image. The circulation data follow the same trends as the data for maximum vorticity. Circulation of the secondary vortex and the descending drop are of the sign opposite to the primary vortex ring. Each are represented by a broken line. The circulation of the vortex drop is, approximately, $20 \%$ of the initial vortex ring circulation. The circulation of the tight vortex created near the neck of the column is, approximately, $3 \%$ of the initial vortex ring circulation, at the last measurement point.

For the high Weber number case shown in Figure 7 , the maximum vorticity is plotted in Figure 10. The data indicate an exponential decrease in the peak vorticity. By the end of the interaction, the peak value of vorticity is reduced to approximately $45 \%$ of the initial value of $450 \mathrm{~s}^{-1}$ measured for the vortex ring. The circulation decrease, however, seems to be linear, as shown in Figure 11. The circulation of the vortex drop created by vortex ring separation from the interface is approximately $55 \%$ of the initial vortex ring circulation.

\section{Conclusions}

The main conclusions of this study are as follows:

- At low Weber number, large amounts of opposite sign vorticity are generated at the interface that slows down and eventually stops the drop motion.

- At low Weber number, vorticity is shed from the vortex ring and generated at the interface in the liquid column below the vortex ring.

- At high Weber number no opposite sign vorticity is found, although the circulation of the vortex ring is reduced by $55 \%$ during the interaction.

\section{Acknowledgements}

This research sponsored by NASA Microgravity Fluid Physics Program, Grant no. NCC3-474.

\section{References}

1. Bernal, L.P., Maksimovic, P., Tounsi, F. \& Tryggvason, G. "An experimental and numerical investigation of drop formation by vortical flows in microgravity," AIAA paper 94-0244, AIAA $32^{\text {nd }}$ Aerospace Sciences Meeting \& Exhibit. Reno, NV. January 10-13, 1994.

2. Glezer, A. (1988) The Formation of Vortex Rings. Phys. Fluids 31: 3532-3542.

3. Maksimovic, P. (1997) "Vortex ring interaction with liquid interfaces: A study of a drop formation process," Ph.D thesis, University of Michigan.

4. Landreth, C.C., and Adrian, R.J. (1988) Electrooptical Image Shifting for Particle Image Velocimetry. Appl. Opt. 27 (20) 4216-4220.

5. Willert, C.E., and Gharib, M. (1991) Digital Particle Image Velocimetry. Exp. Fluids 10: 181-193.

6. Adrian, R.J. (1986) Image Shifting Technique to Resolve Directional Ambiguity in Double-Pulsed Velocimetry. Appl. Optics 25: 3855-2858.

7 Adrian, R.J. (1991) Particle-Imaging Techniques for Experimental Fluid Mechanics. Annu. Rev. Fluid Mech. 23: 261-304.

8 McDougall, T.J. (1979) On the Elimination of Refractive-Index Variations in Turbulent DensityStratified Liquid Flows. J. Fluid Mech. 93: 83-96. 
9. Hannoun, I.A., Fernando, H.J.S., and List, E.J. (1988) Turbulent Structure Near a Sharp Density Interface. J. Fluid Mech. 189: 189-209.

10. Alahyari, A., and Longmire, E.K. (1994) Particle Image Velocimetry in a Variable Density Flow: Application to a Dynamically Evolving Microburst. Exp. Fluids 17: 434-440.

11. Keane, R.D., and Adrian R.A. (1990) Optimization of Particle Image Velocimeters. Part I: Double Pulsed Systems. Meas. Sci. Technol. 1: 1202-1215.

12. Prasad, A.K., and Adrian, R.J. (1993) Stereoscopic Particle Image Velocimetry Applied to Liquid Flows. Exp. Fluids 15: 49-60.

13. Oschwald, M., Bechle, S., and Welke, S. (1995) Systematic Errors in PIV by Realizing Velocity Offsets with the Rotating Mirror. Exp. Fluids 18: 329-334.
14.Zhang, Z., and Eisele, K. (1995) The TwoDimensional Velocity Shift Caused by the Use of a Rotating Mirror in PIV Flow Field Measurements. Exp. Fluids 20: 106-111.

15. Luff, J.D., Rompage, A.M., Linne, M.A., and Hertzberg, J.R. (1996) Uncertainties Associated with the Post-Processing of 2-D Particle Image Velocimetry (PIV) Velocity data of Unsteady Flow Fields. Western States Section, the Combustion Institute. Spring Meeting. Tempe, AZ. Paper WSS 96S-005.

16. Karri, S.B.R., and Mathur, V.K. (1988) Measurement of Interfacial Tension of Immiscible Liquids of Equal Density. AlChe Journal 34: 155 157.

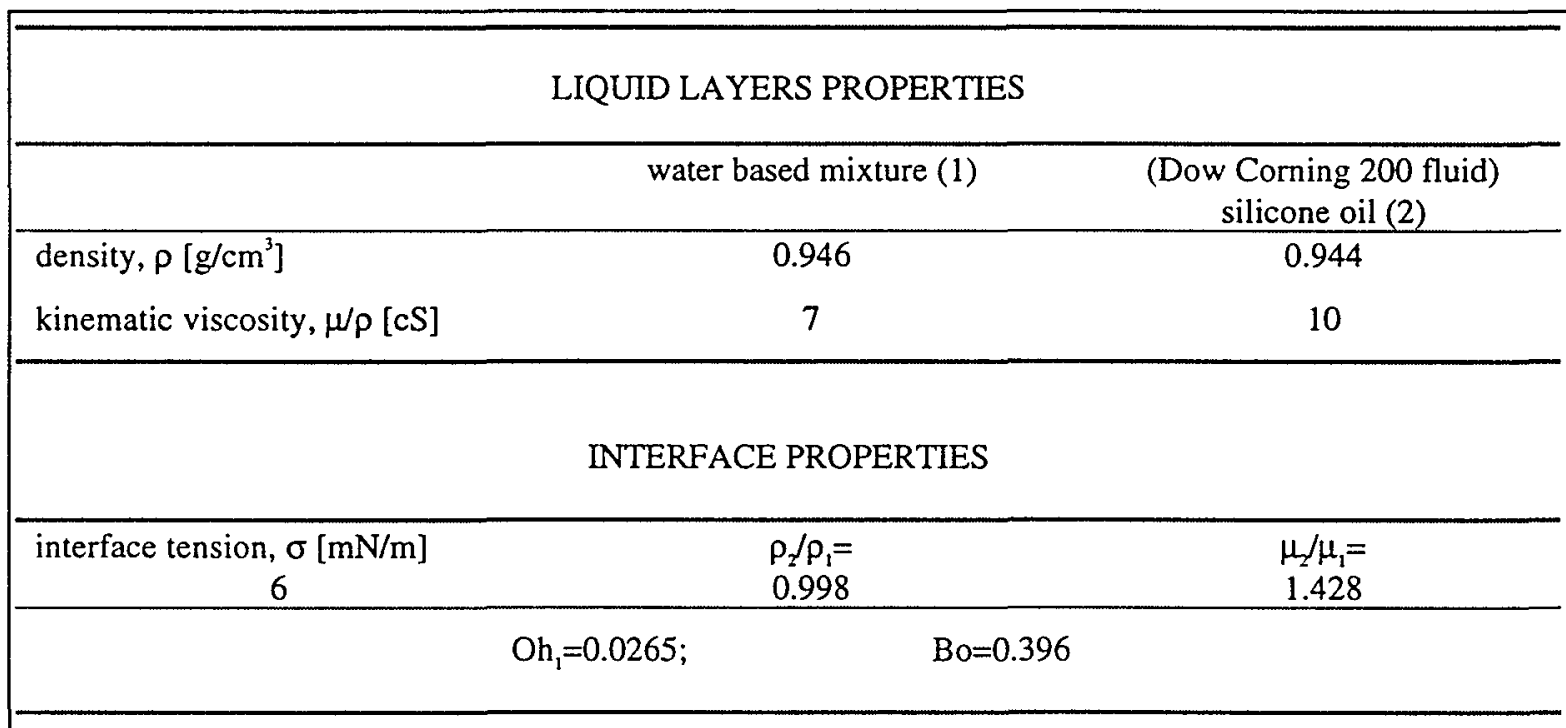

Table 1: $\quad$ Physical properties of the immiscible liquid pair used in PIV measurements. 

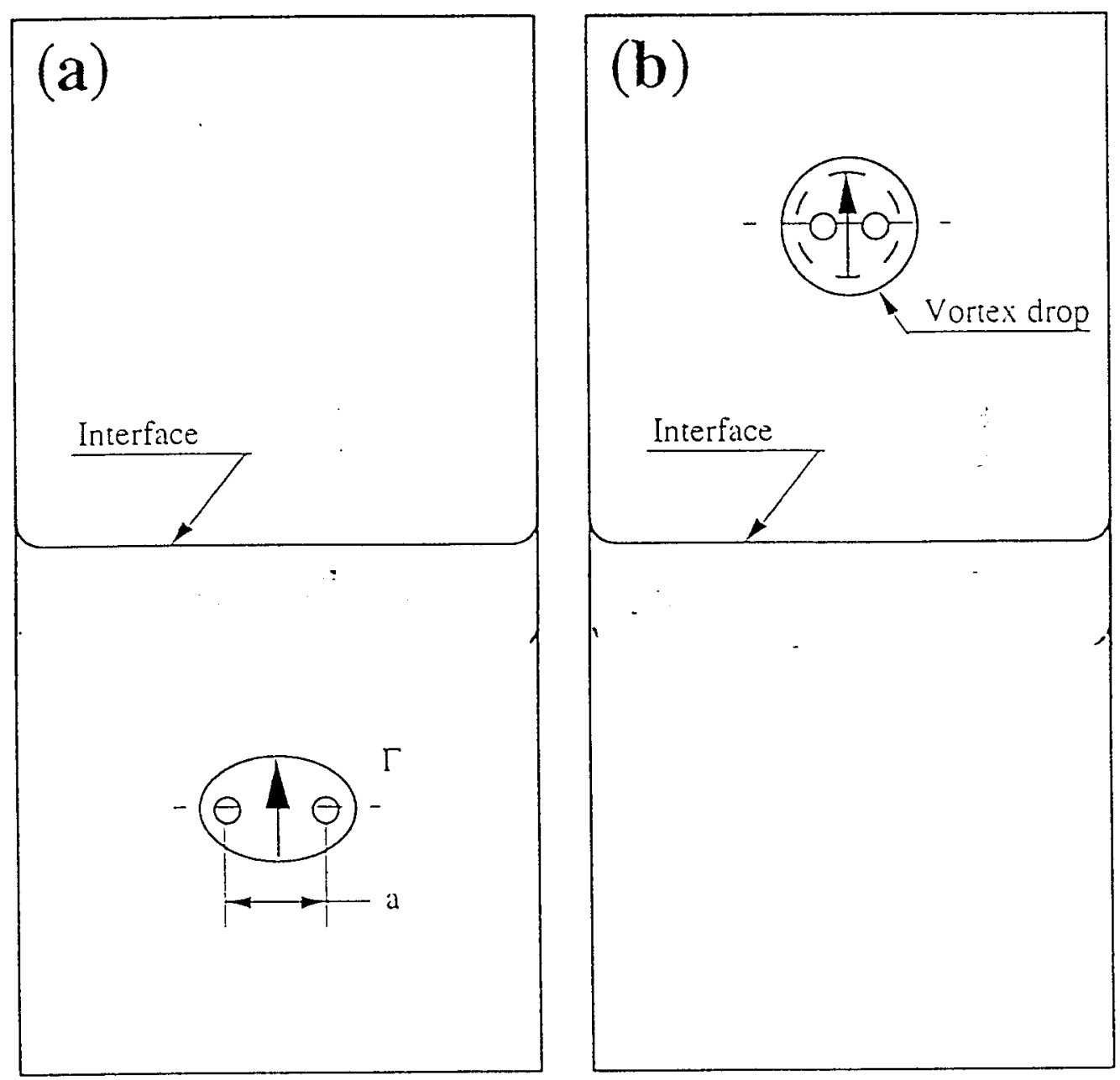

Figure 1 Schematic diagram of the interaction of a vortex ring with an immiscible liquid interface. (a) Before the interaction (b) After the interaction. 


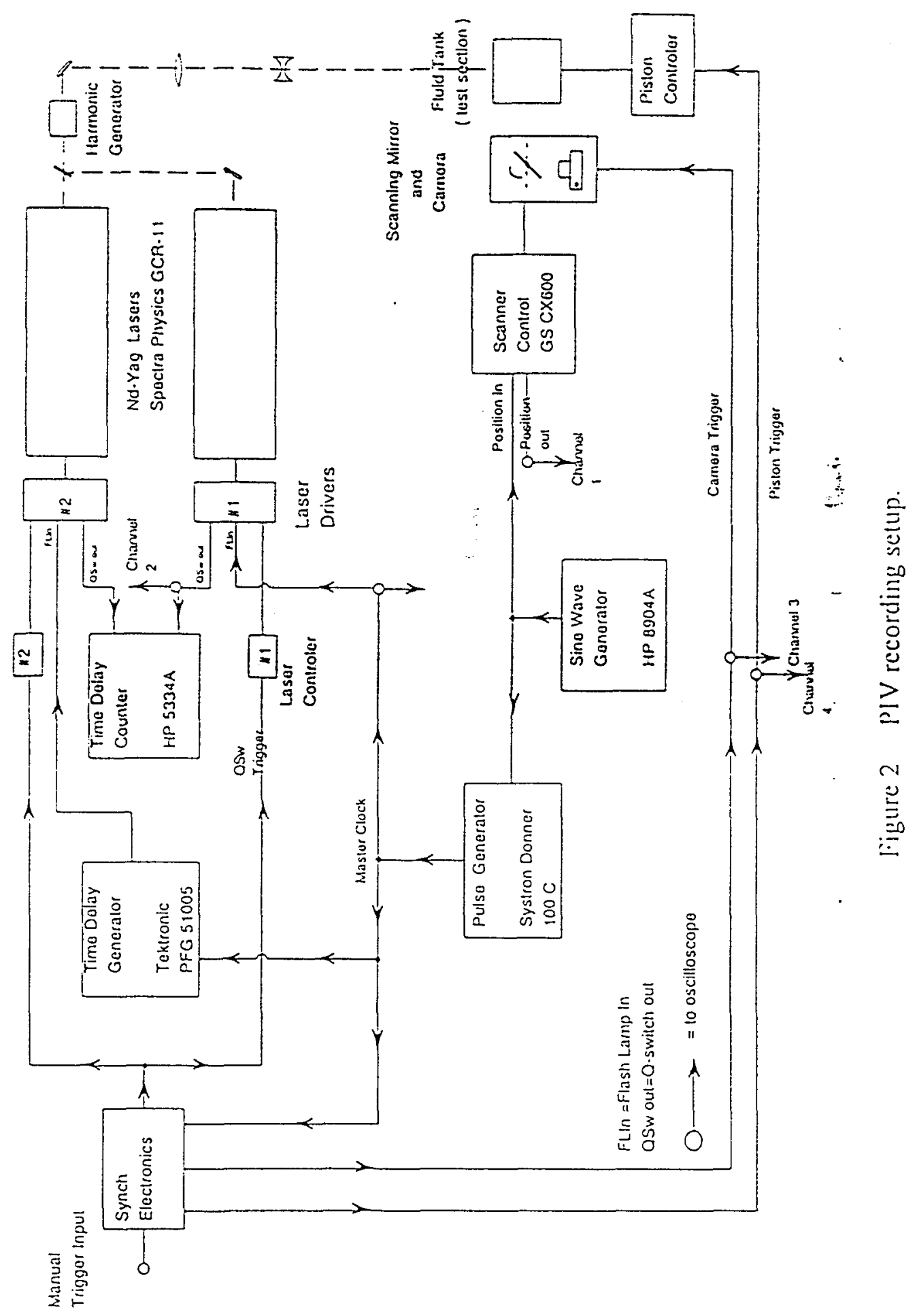


Copyright $@ 1998$, American Institute of Aeronautics and Astronautics, Inc.

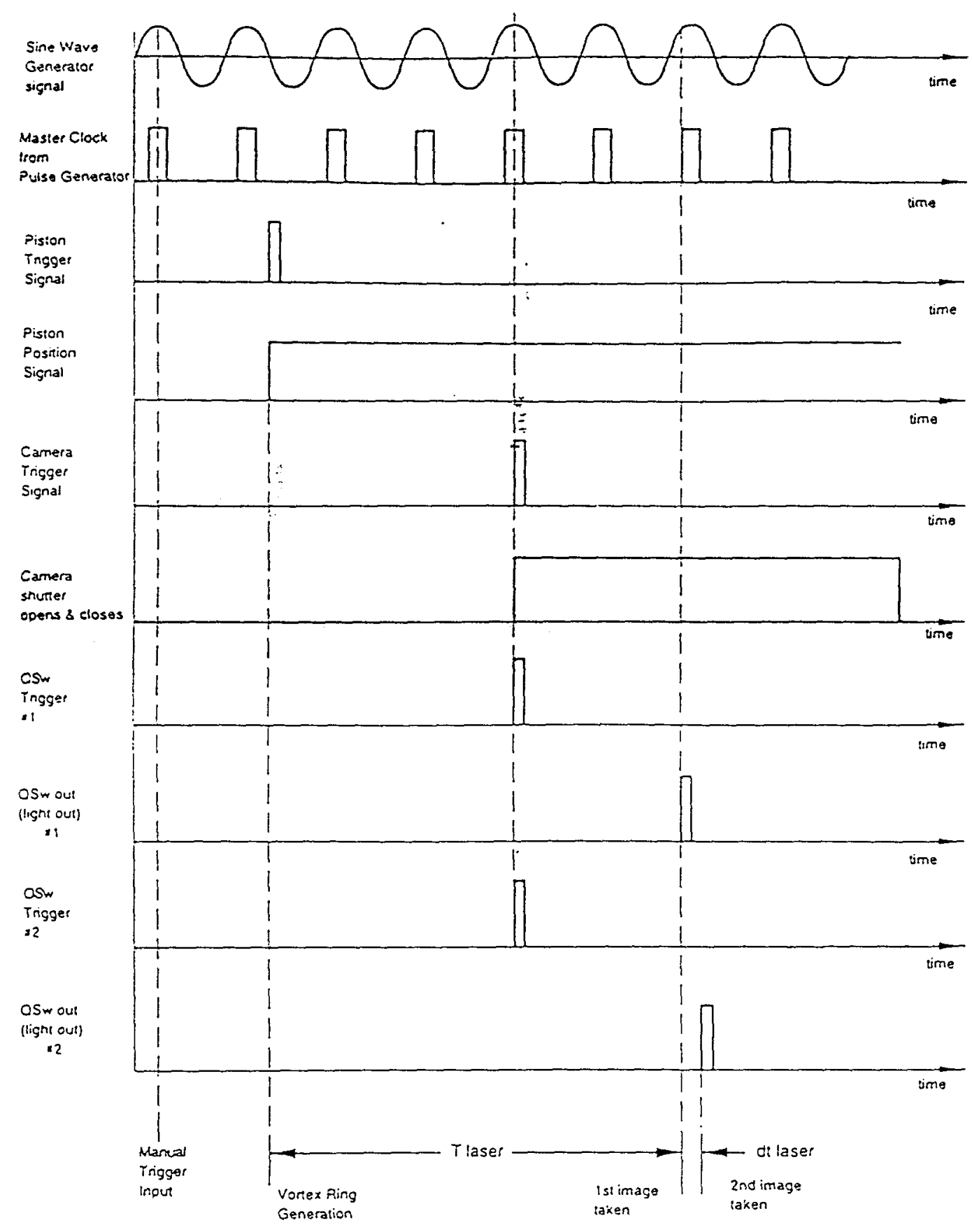

Figure 3 Signal synchronization of the PIV electronics. 

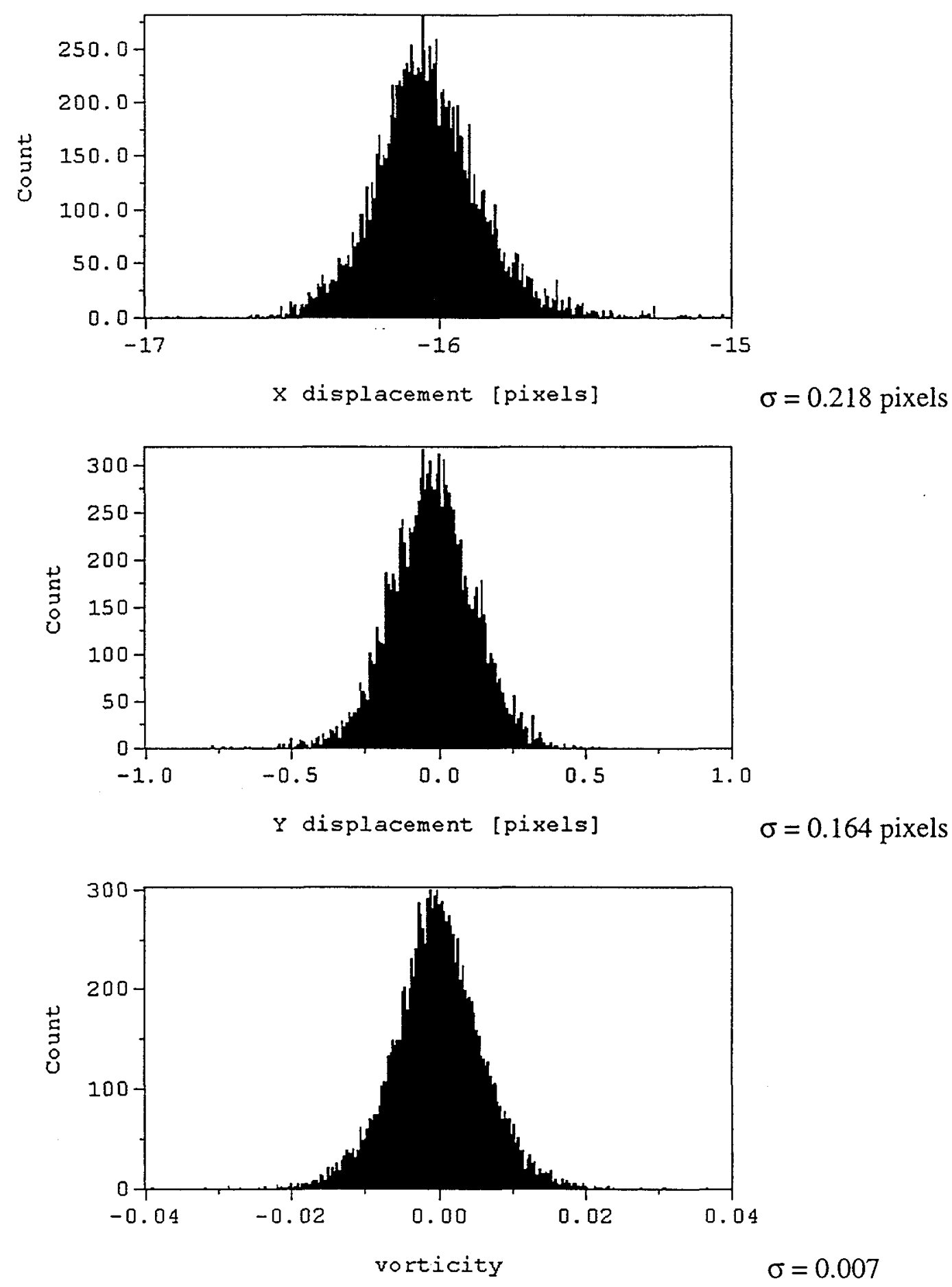

Figure 4 Histogram of the $x$-component displacement, $y$ - component displacement, and vorticity measured with liquid at rest. Two liquid layers. 

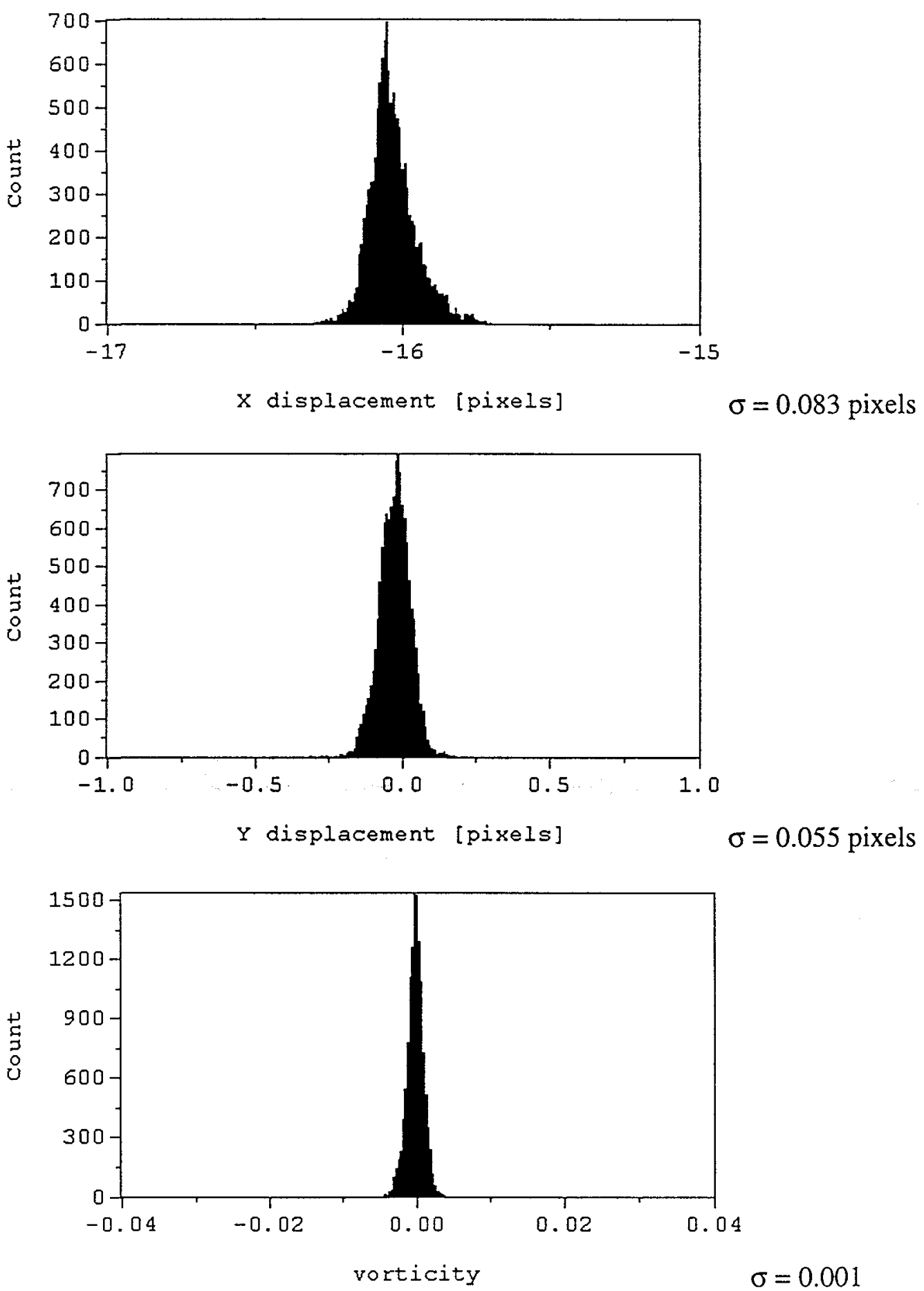

Figure 5 Histogram of the filtered particle displacement and vorticity for the data shown in Figure 4. 
a) $t=0.508 \mathrm{~s}$
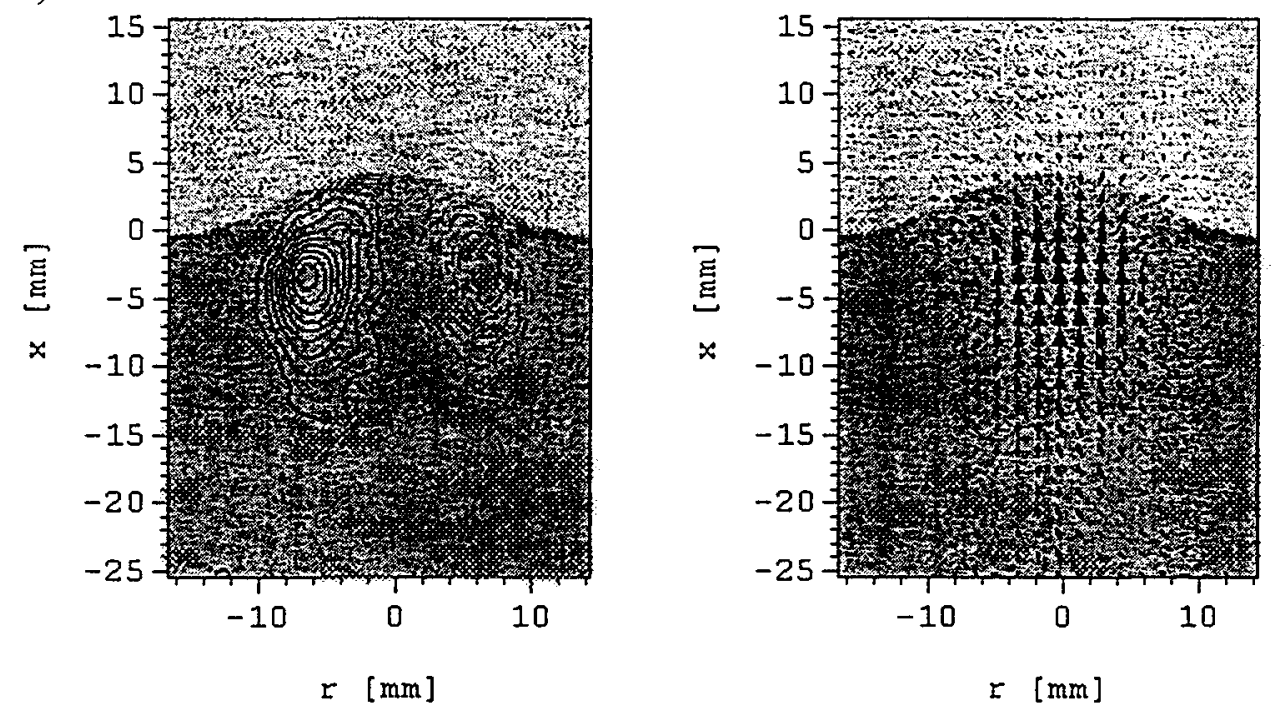

b) $t=0.613 \mathrm{~s}$
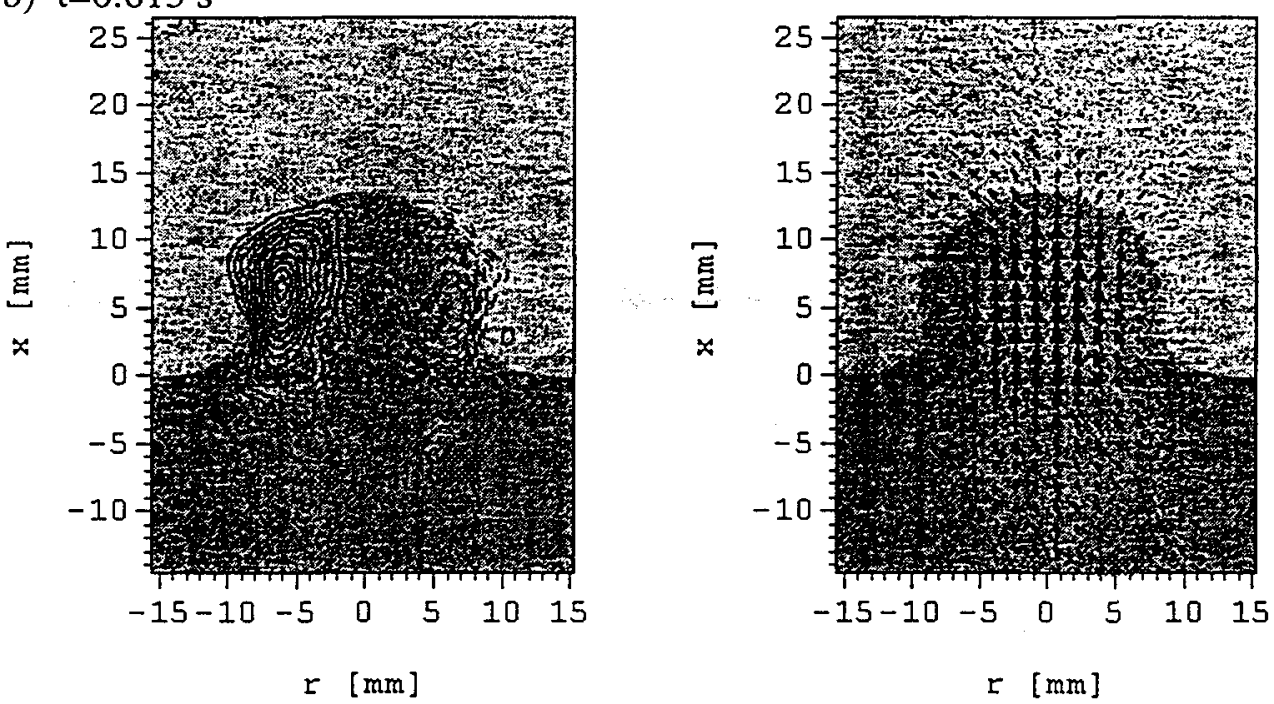

Figure 6 PIV velocity and vorticity field for $R e=420, W e=125$. The vorticity contours and velocity vectors are overlaid on the actual PIV image. The starting contour values $\left[\mathrm{s}^{-1}\right]$ and the contour increment $\left[\mathrm{s}^{-1}\right]$ of vorticity are: a) $\pm 6.6,6$; b) $\pm 6.5,4.7$; c) $\pm 5,3.5$; d) $\pm 2.5,3$; e) $\pm 1.5,1.7$; f $\pm 1.5,1.4$; g) $\pm 0.8,0.8$; $)$ $\pm 1,1.2$; i) $\pm 0.9,0.9$; j) $\pm 1.2,0.9$; k) $\pm 1,1 ; \mathrm{l}) \pm 0.7,1.1$. 
Copyright @ 1998, American Institute of Aeronautics and Astronautics, Inc.

c) $t=0.739 \mathrm{~s}$
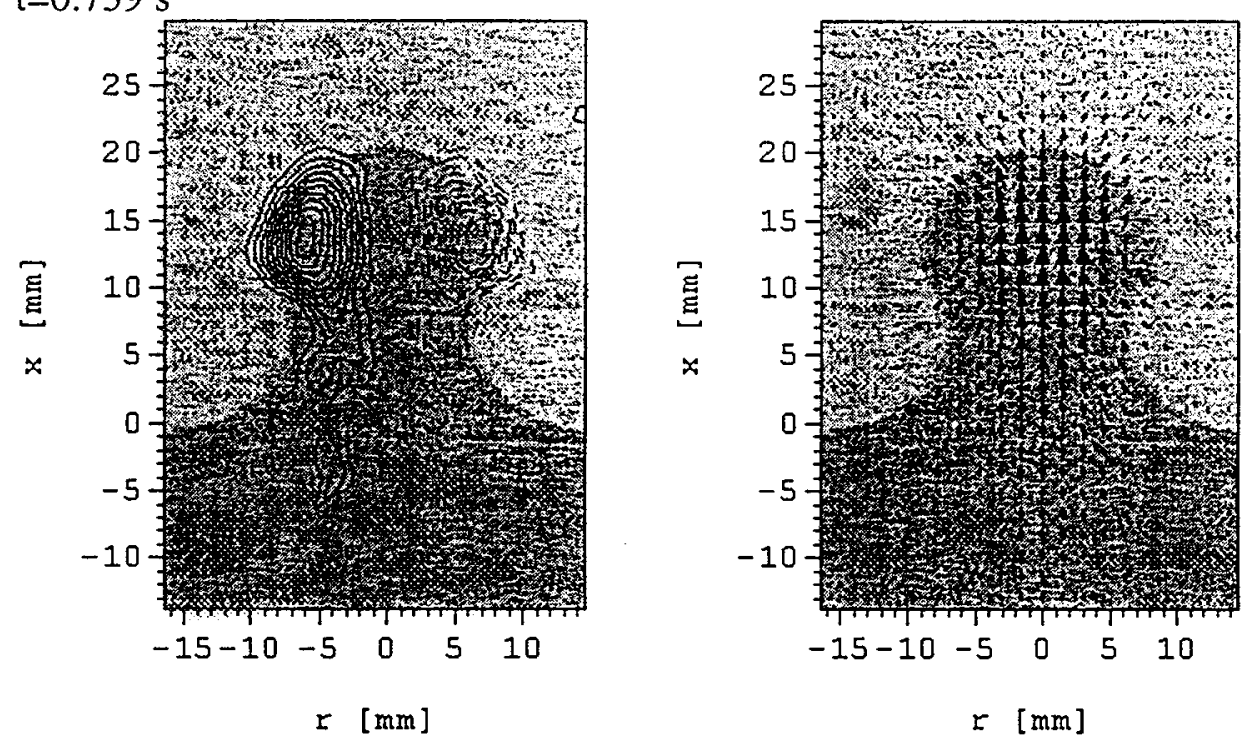

d) $t=0.857 \mathrm{~s}$
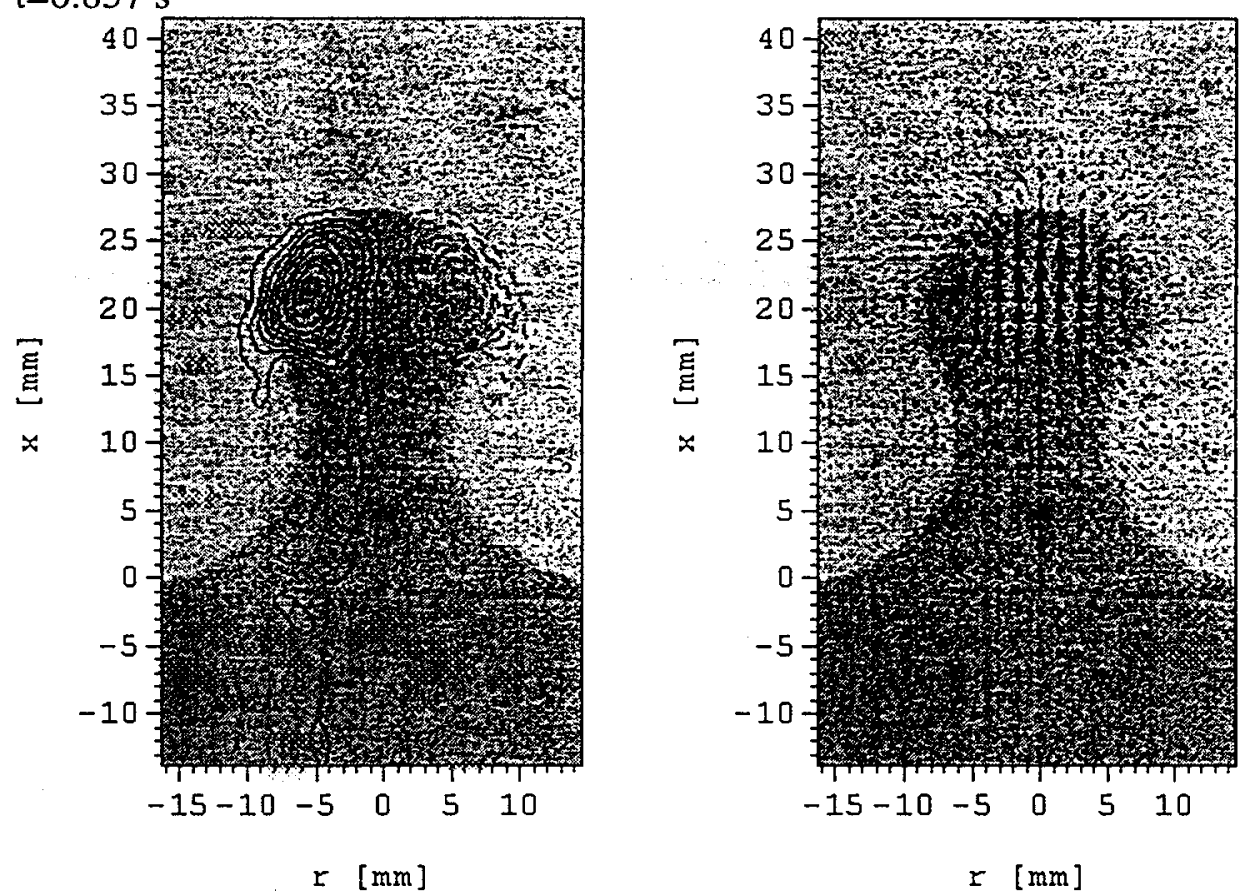

Figure 6 (Continued.) 
e) $t=1.052 \mathrm{~s}$

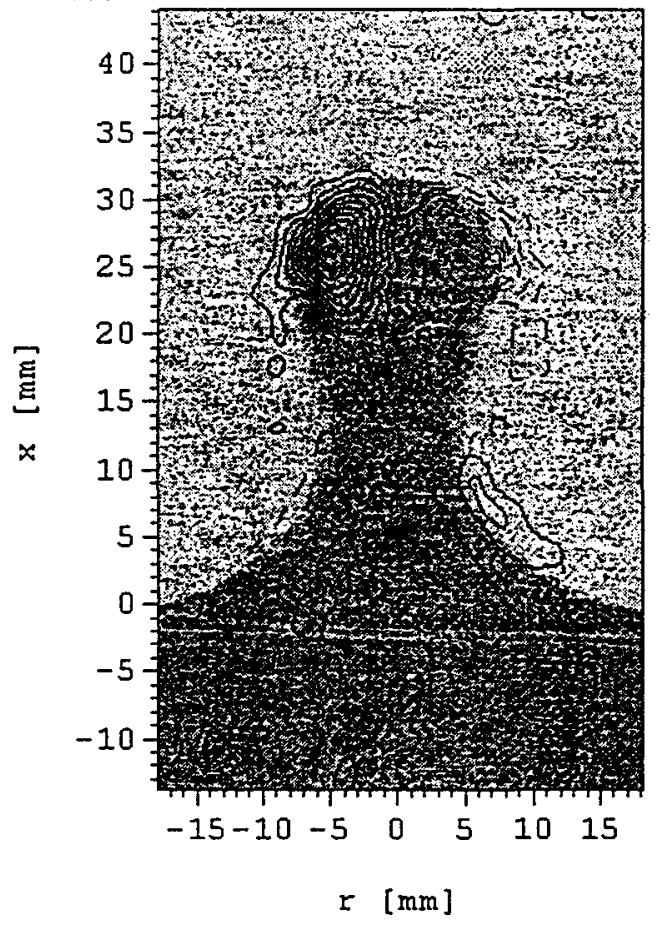

f) $t=1.170 \mathrm{~s}$

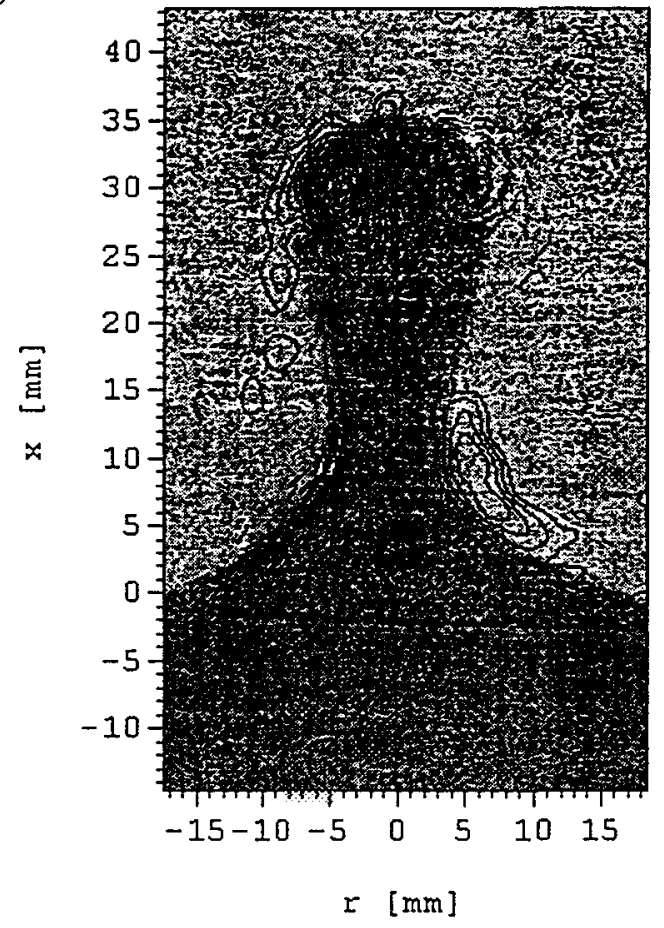

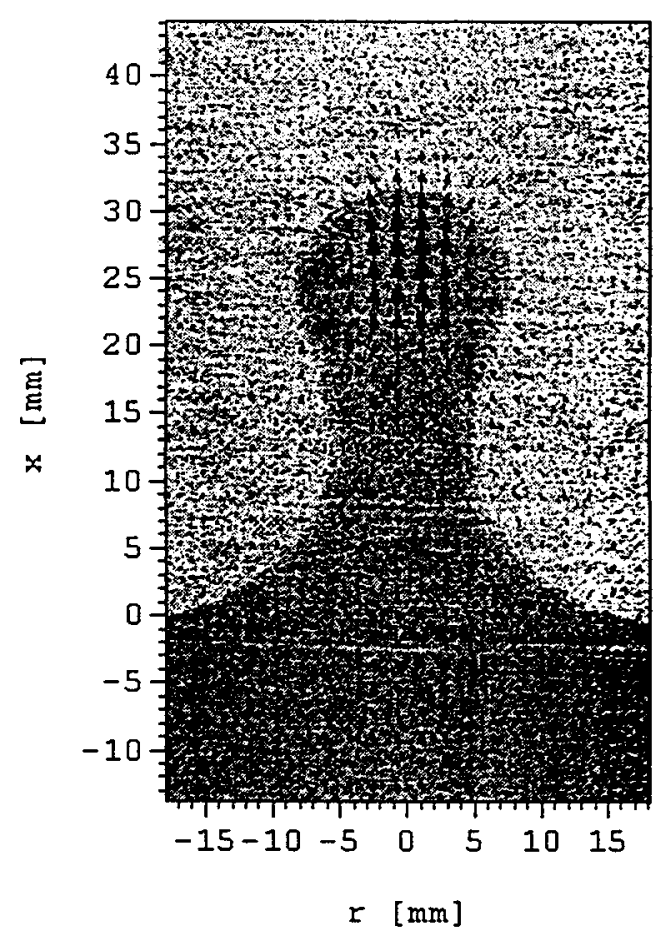

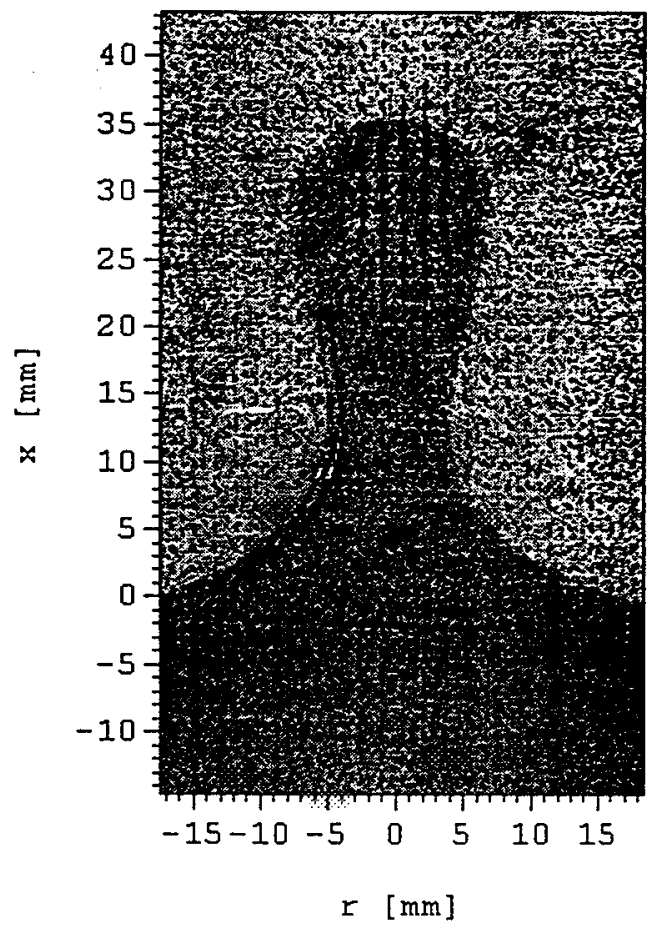

Figure 6 (Continued.) 
g) $\mathrm{t}=1.319 \mathrm{~s}$

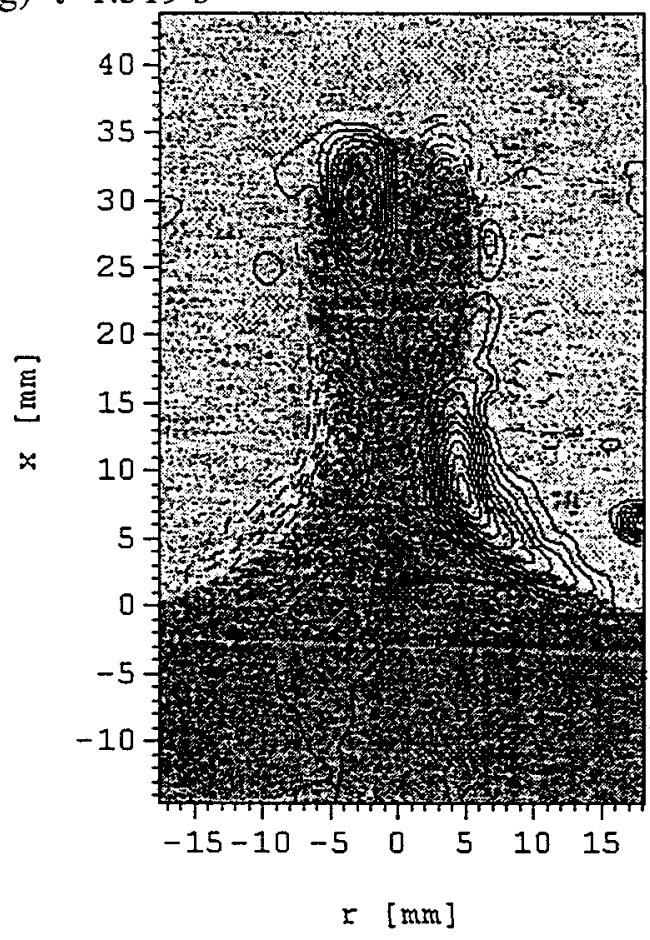

h) $t=1.477 \mathrm{~s}$

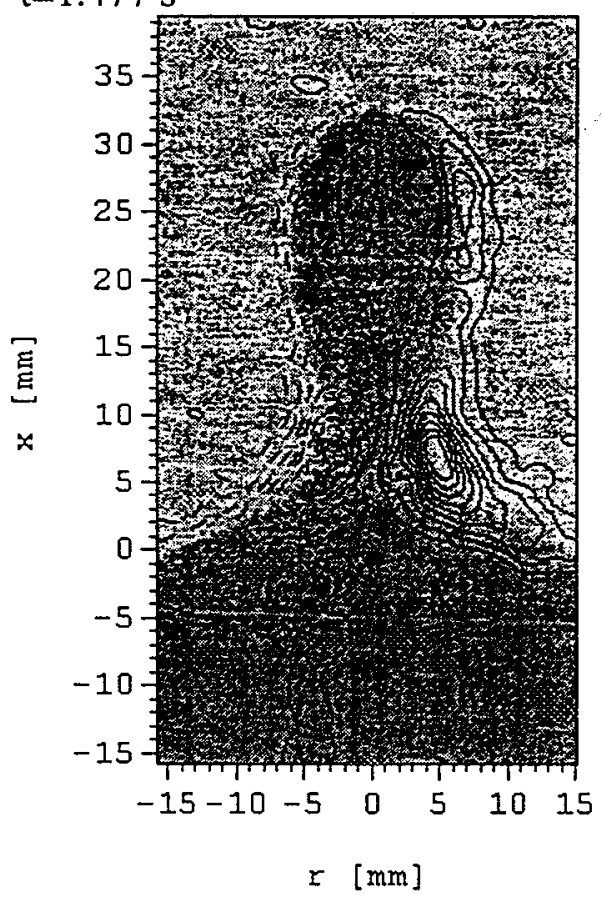

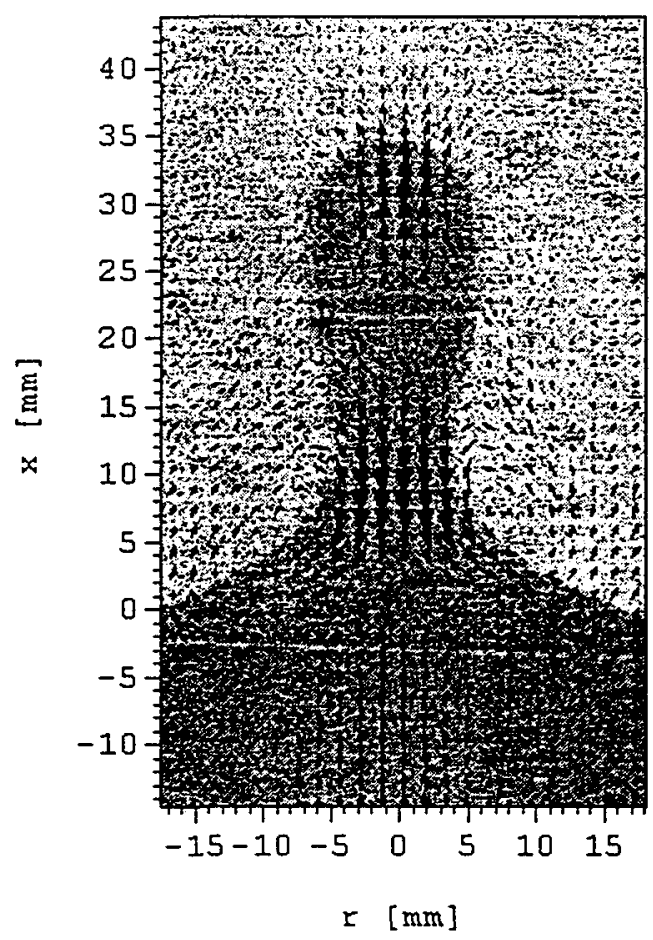

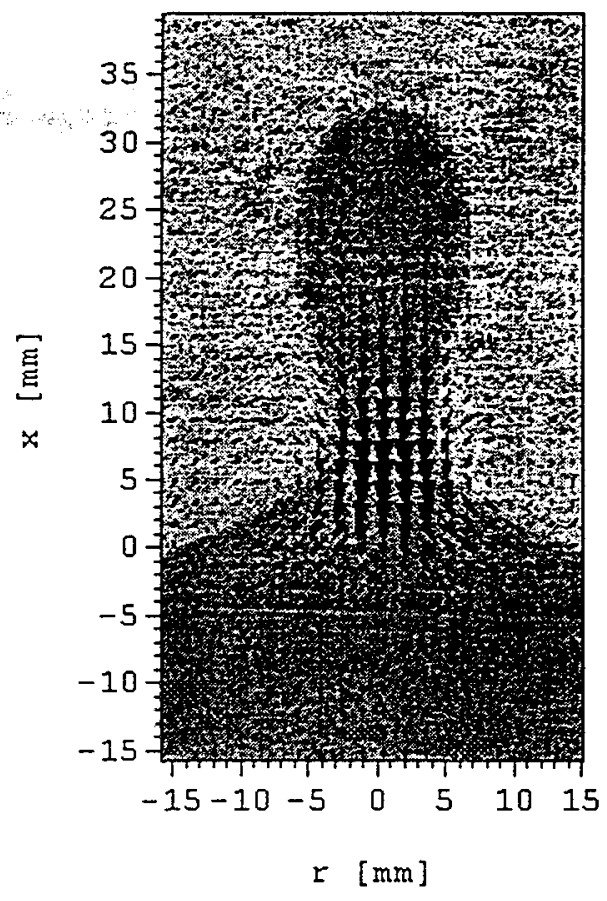

Figure 6 (Continued.) 
i) $t=1.723 \mathrm{~s}$

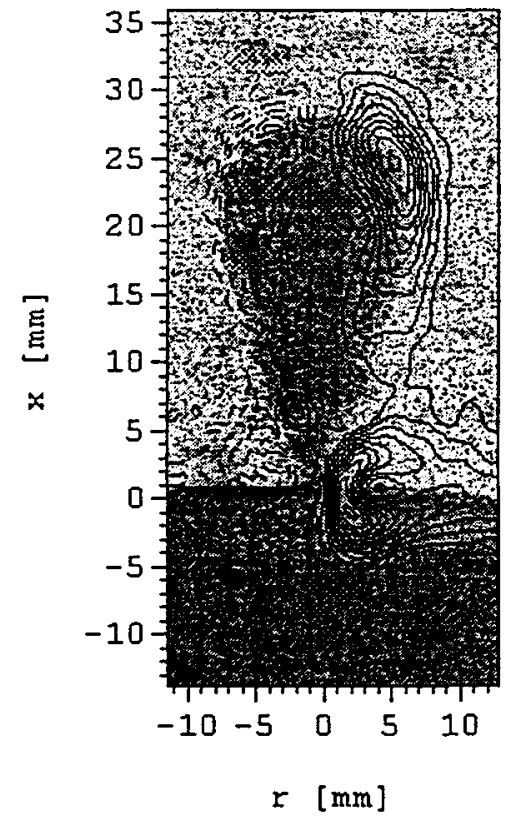

j) $t=1.726 \mathrm{~s}$

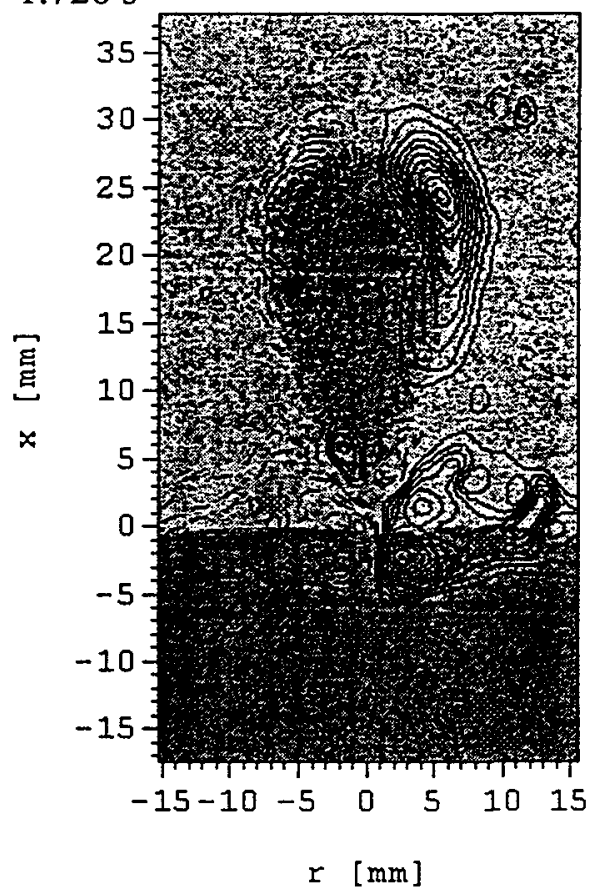

Figure 6 (Continued.)
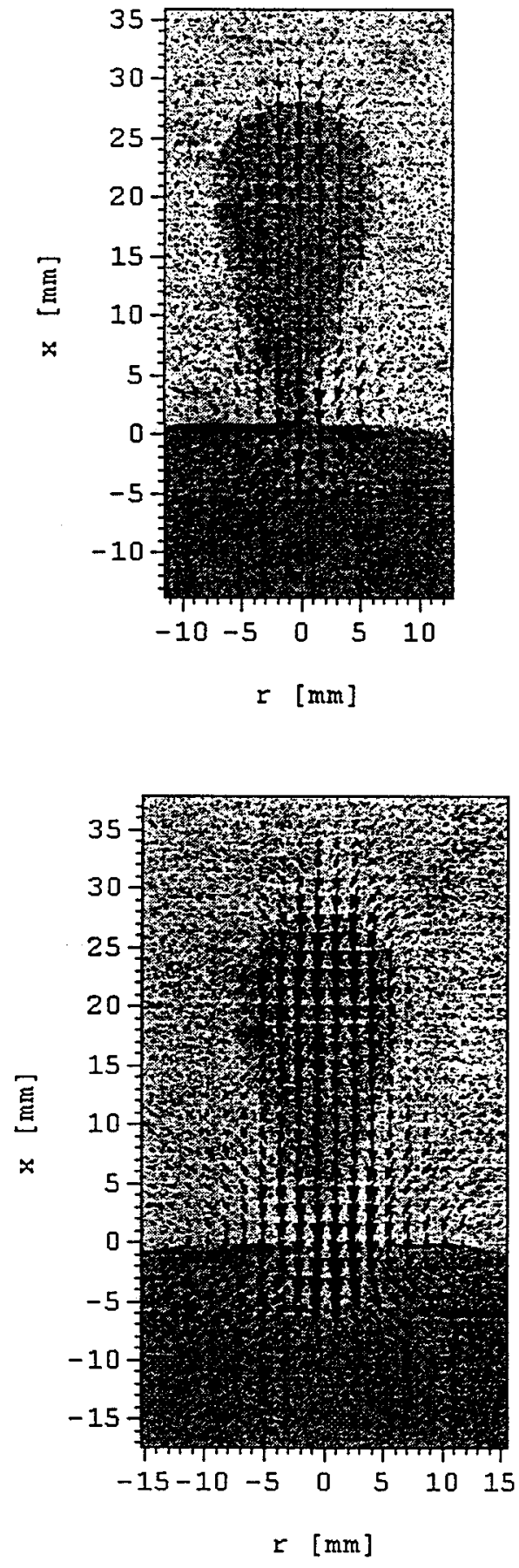
Copyright (C) 1998, American Institute of Aeronautics and Astronautics, Inc.

k) $t=1.879 \mathrm{~s}$
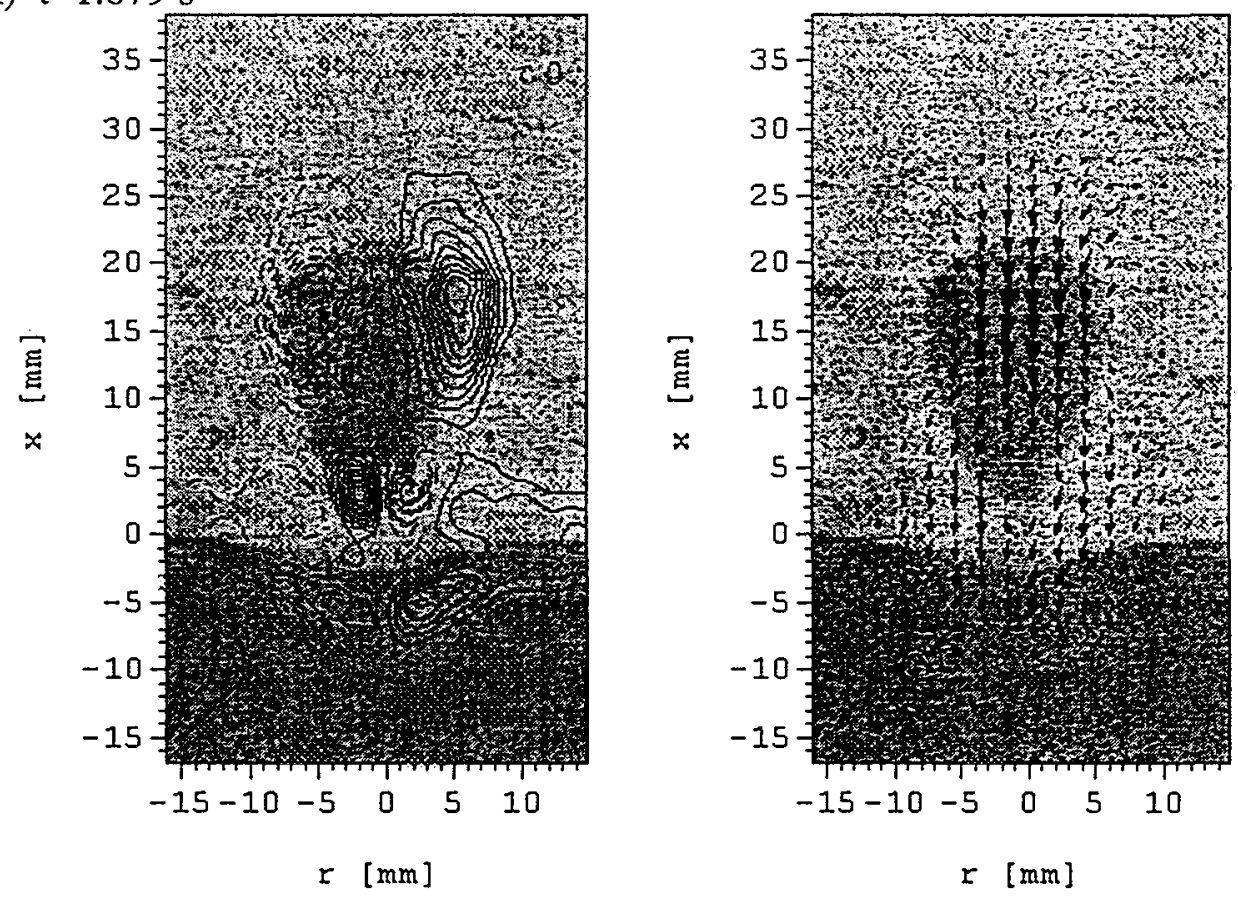

1) $\mathrm{t}=2.102 \mathrm{~s}$
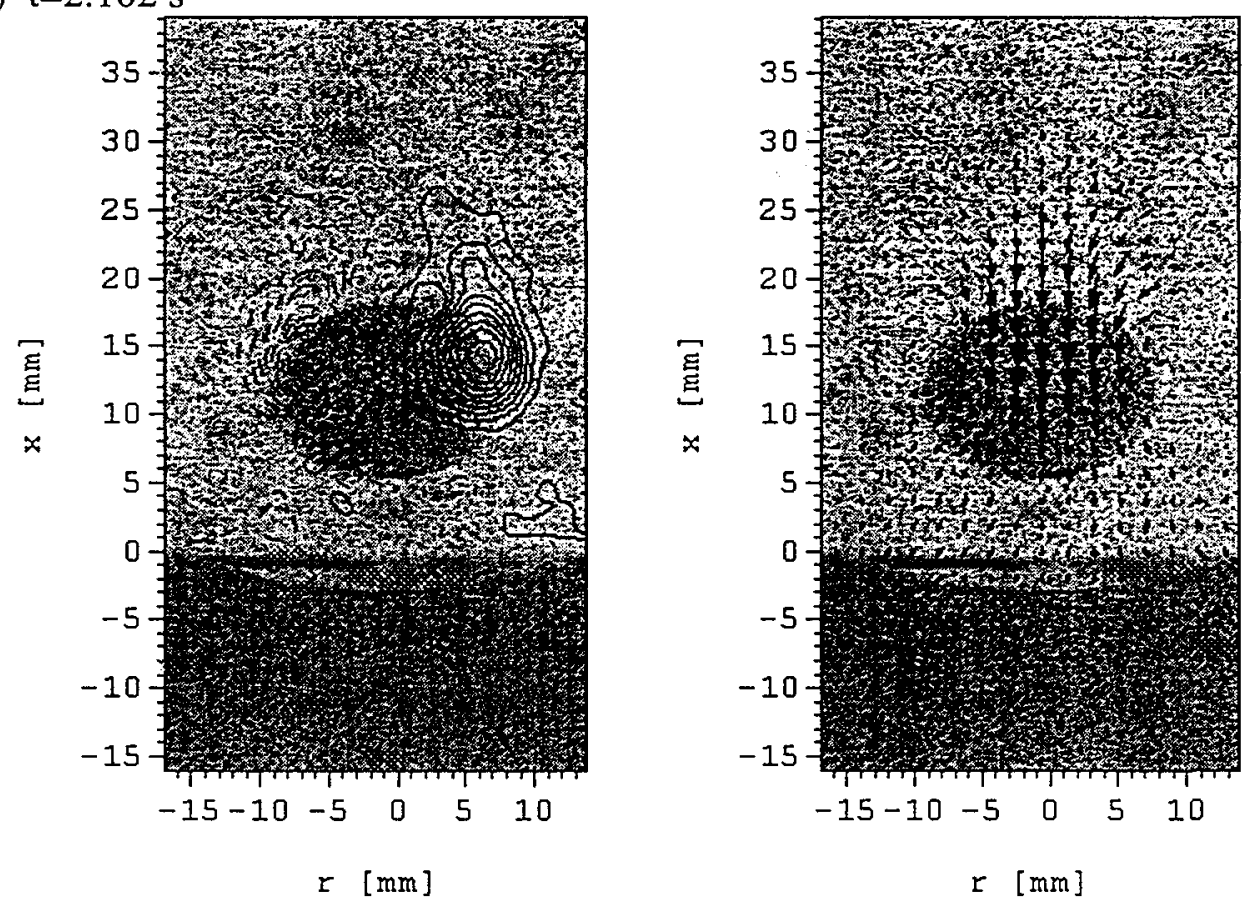

Figure 6 (Concluded.) 
a) $t=0.234 \mathrm{~s}$
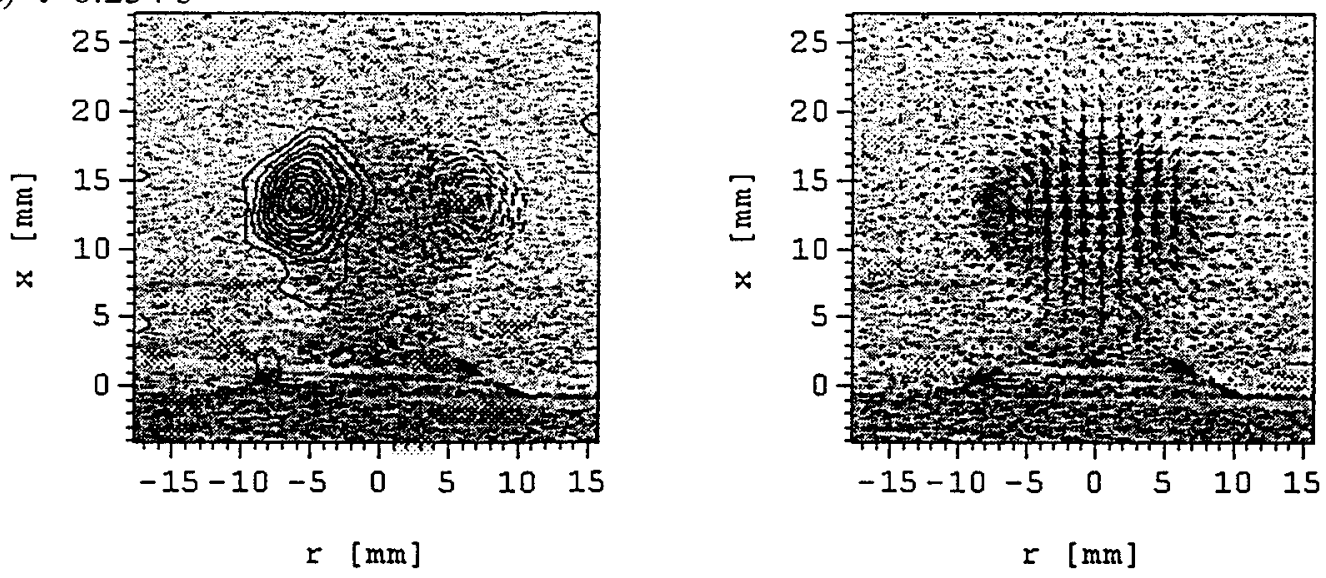

b) $t=0.274 \mathrm{~s}$
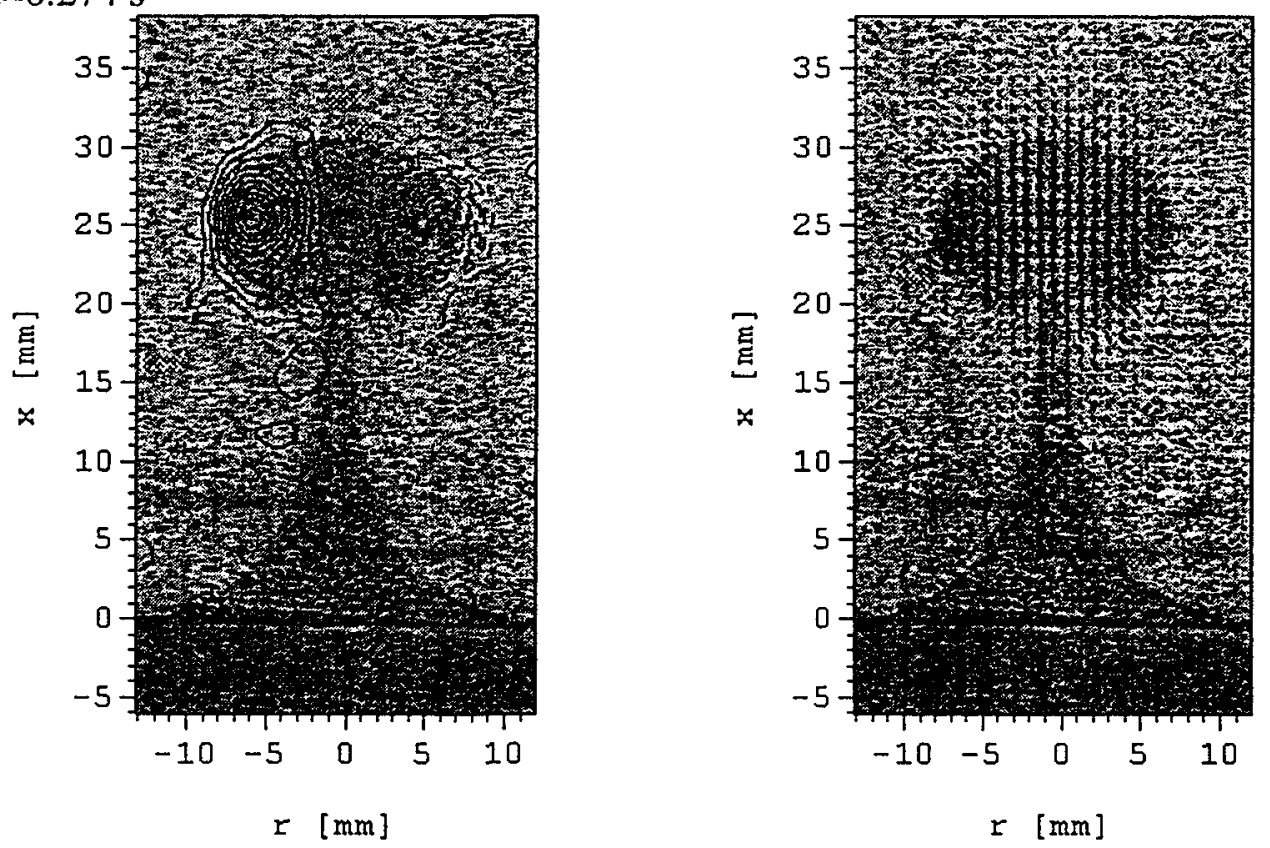

Figure 7 PIV velocity and vorticity field for $\mathrm{Re}=1250, \mathrm{We}=1085$. The vorticity contours and velocity vectors are overlaid on the actual PIV image. The starting contour values $\left[\mathrm{s}^{-1}\right]$ and the contour increment $\left[\mathrm{s}^{-}\right.$ ${ }^{1}$ ] of vorticity are: a) $\pm 27,25$; b) $\pm 20,22$; c) $\pm 18.5,18.5$. 
c) $t=0.351 \mathrm{~s}$
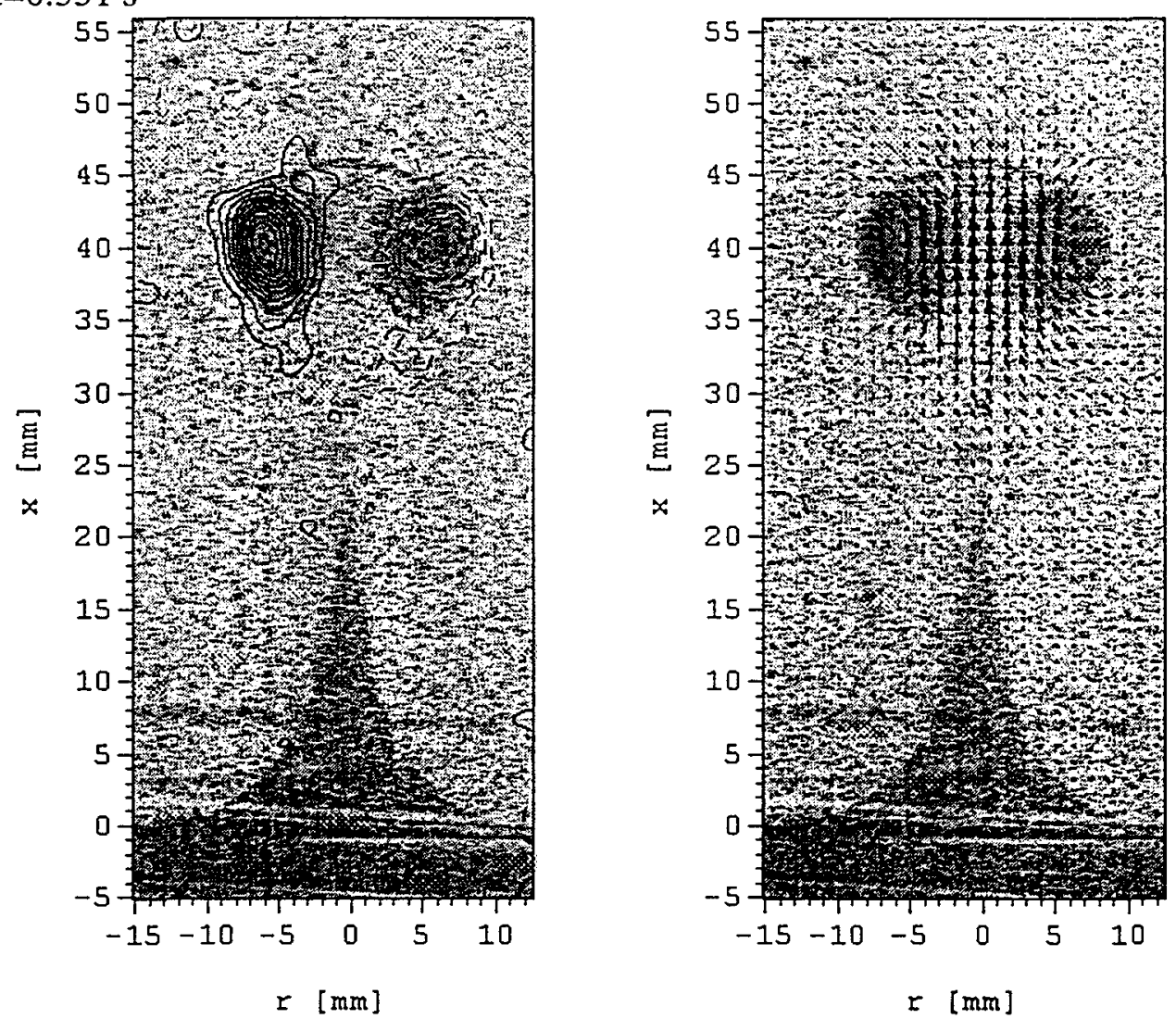

Figure 7 (Concluded.)

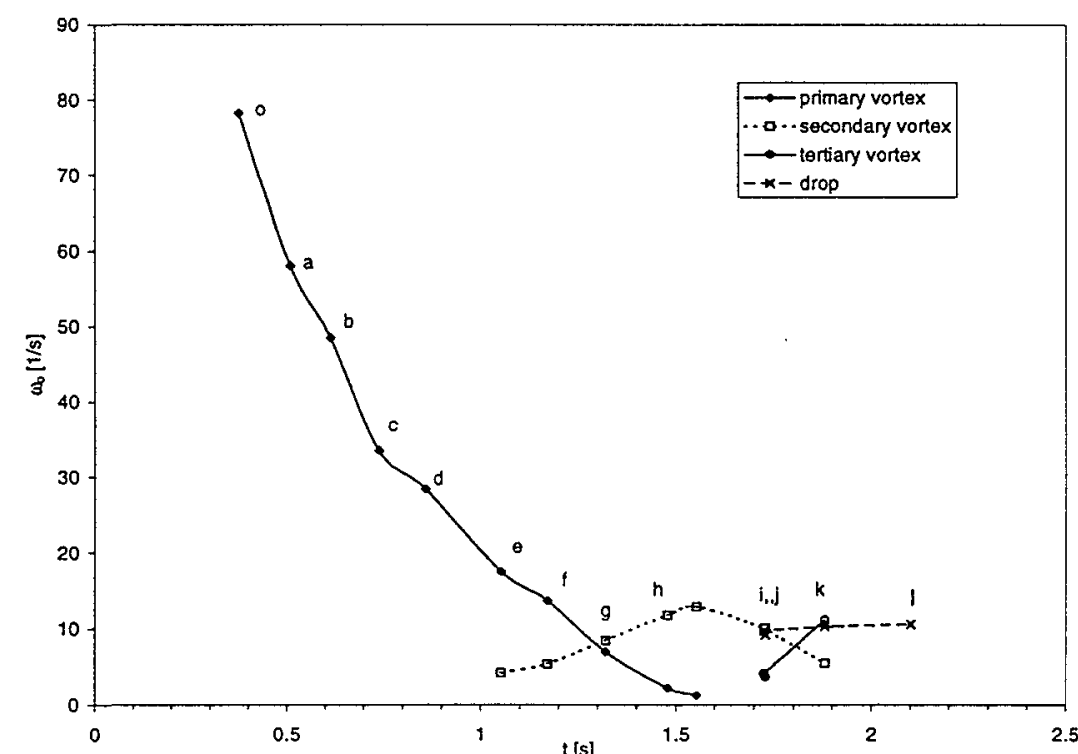

Figure 8 Time evolution of the maximum vorticity for the vortices formed at $\mathrm{Re}=420, \mathrm{We}=125$, shown in Figure 5.3. 


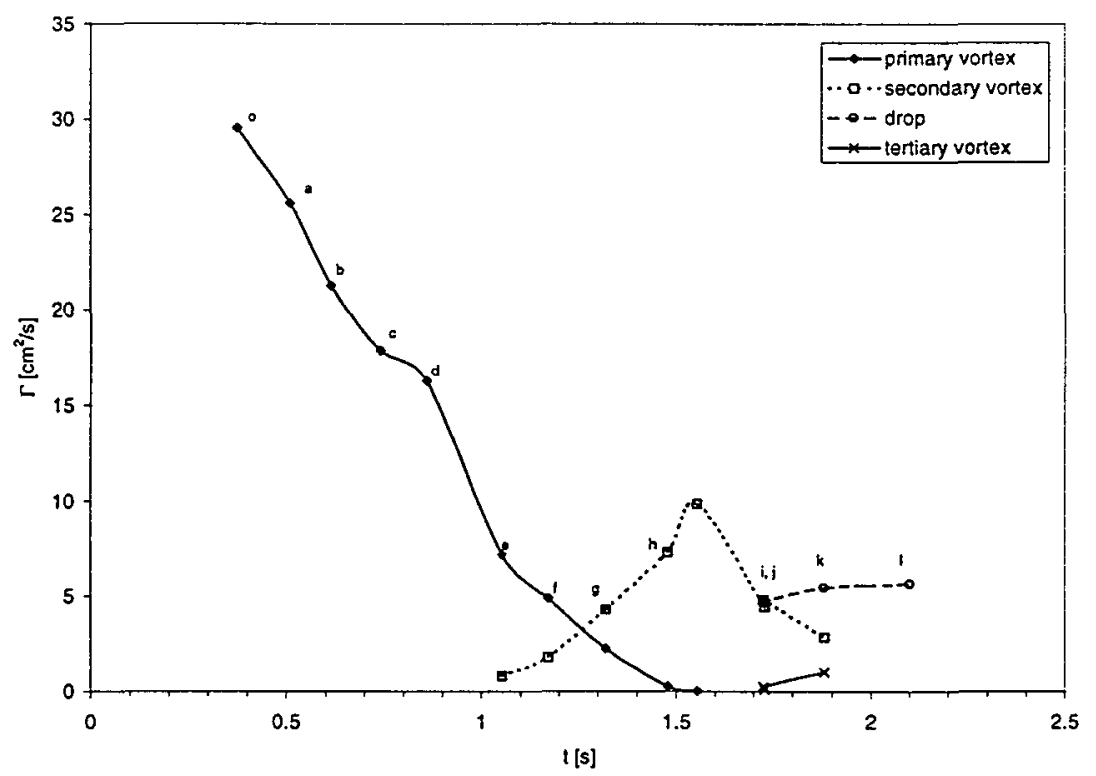

Figure 9. Time evolution of the circulation for the vortices formed at $\mathrm{Re}=420, \mathrm{We}=125$, shown in Figure 5.3.

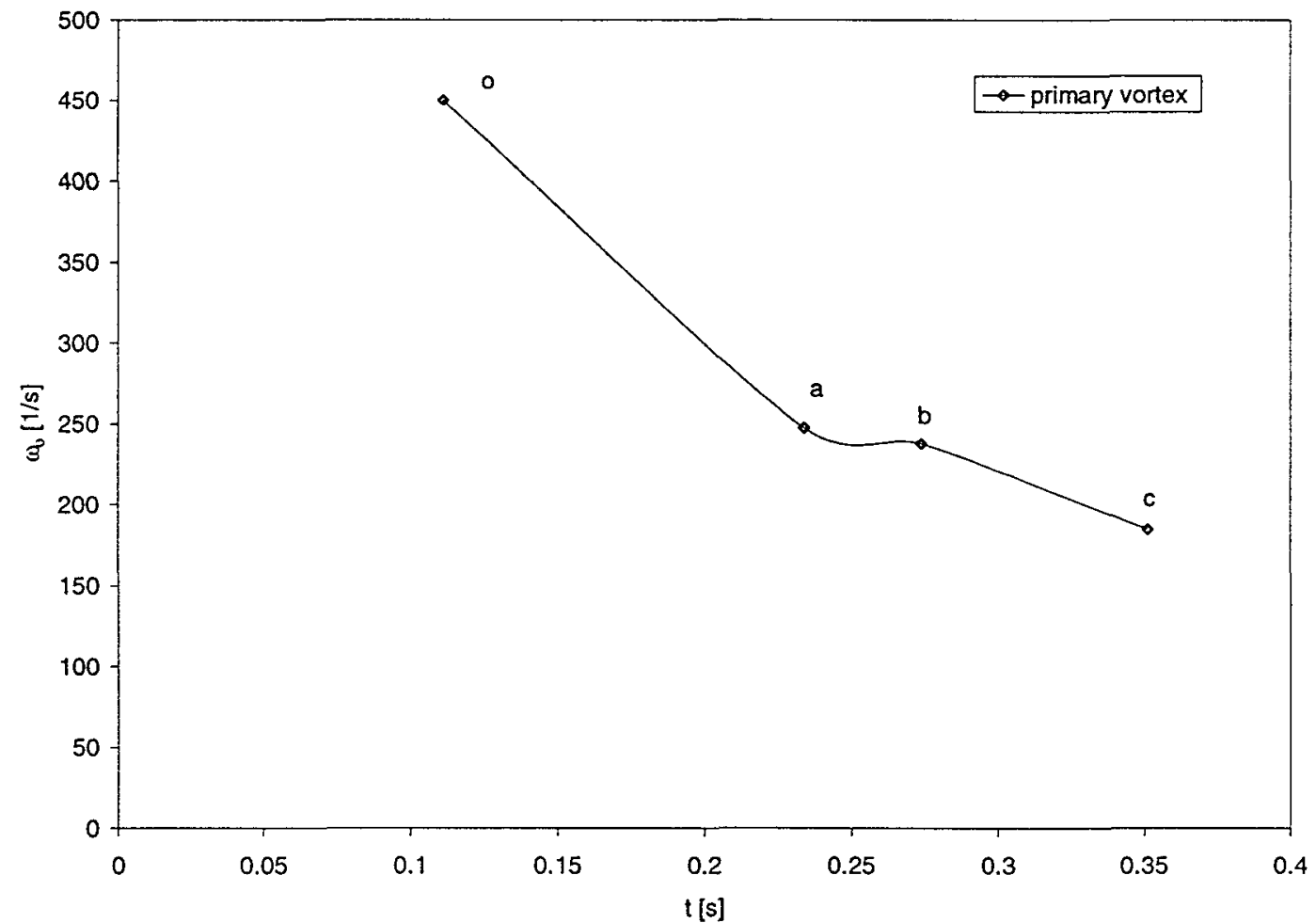

Figure 10 Time evolution of the maximum vorticity for the vortices formed at $R e=1250$, We $=1085$, shown in Figure 5.11. 
Copyright (C) 1998, American Institute of Aeronautics and Astronautics, Inc.

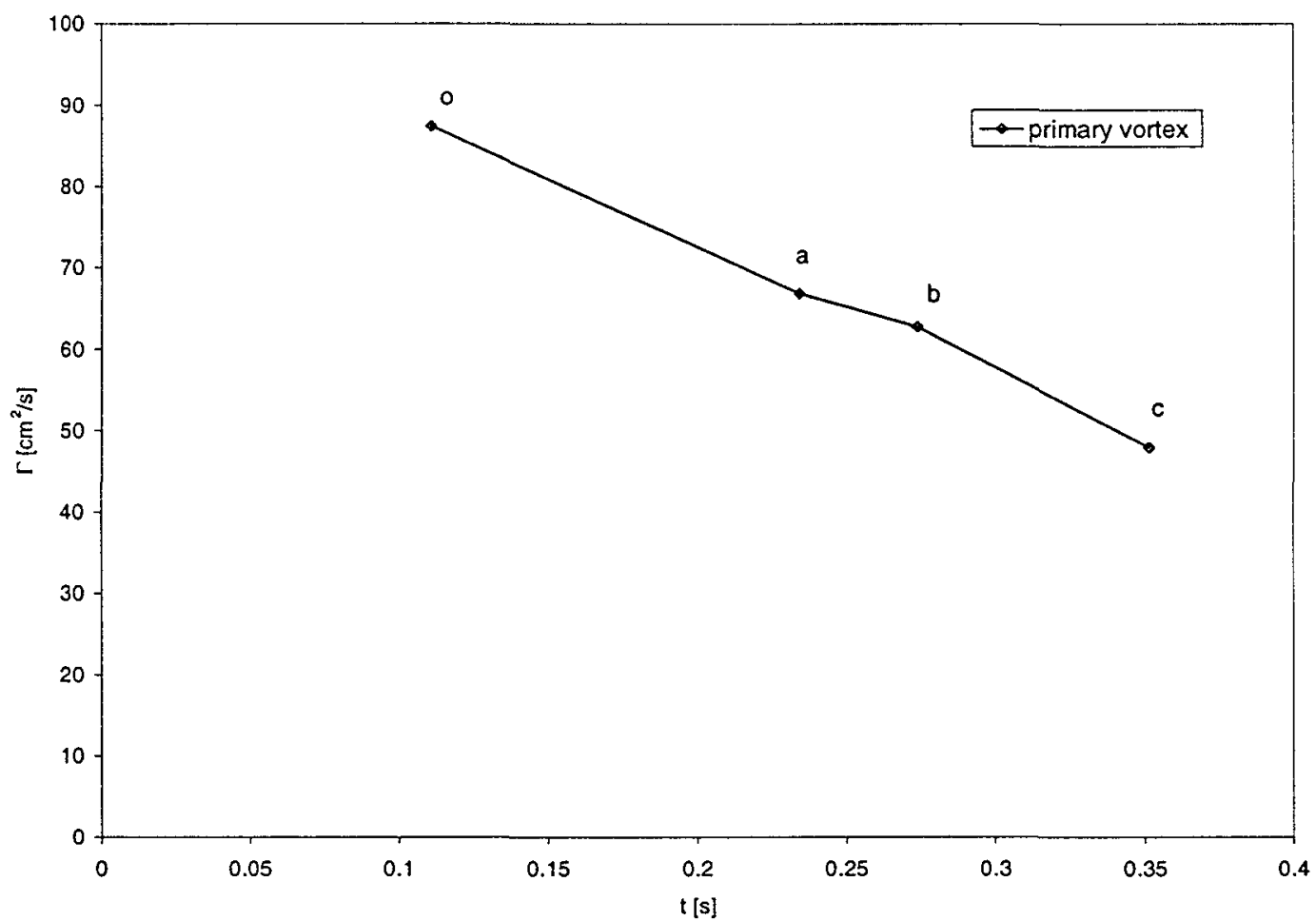

Figure 11 Time evolution of the circulation for the vortices formed at $\operatorname{Re}=1250, \mathrm{We}=1085$, shown in Figure 5.11 . 